\title{
Flots et series de Taylor stochastiques
}

\author{
Gérard Ben Arous \\ Centre de Mathématiques appliquées, Ecole Normale Supérieure, 45 rue d'Ulm, \\ F-75230 Paris Cedex 05 France
}

\begin{abstract}
Summary. We study the expansion of the solution of a stochastic differential equation as an (infinite) sum of iterated stochastic (Stratonovitch) integrals. This enables us to give a universal and explicit formula for any invariant diffusion on a Lie group in terms of Lie brackets, as well as a universal and explicit formula for the brownian motion on a Riemannian manifold in terms of derivatives of the curvature tensor. The first of these formulae contains, and extends to the non nilpotent case, the results of Doss [6], Sussmann [17], Yamato [18], Fliess and Normand-Cyrot [7], Krener and Lobry [19] and Kunita [11] on the representation of solutions of stochastic differential equations.
\end{abstract}

\section{Introduction}

La solution d'une équation différentielle stochastique à coefficients infiniment differentiables n'est en général qu'une fonction mesurable du mouvement brownien qui définit l'équation stochastique. Si les champs de vecteurs qui définisent l'équation commutent (i.e. leurs crochets de Lie sont nuls) alors Doss [6] et Sussmann [17] ont montré qu'en fait la solution de l'équation stochastique est une fonction continue du mouvement brownien; cette fonction continue étant donnée par le flot d'une équation différentielle ordinaire. Nous allons ici étudier l'application «solution» qui au mouvement brownien fait correspondre la solution de l'équation stochastique; plus précisément le but de cet article est d'étudier, en temps petit, le flot défini par une équation différentielle stochastique en montrant le role de la formule de Taylor stochastique par $\mathrm{R}$. Azencott [1], J.-M. Bismut [4], E. Platen [16].

Sous le nom de paramétrix trajectorielle, un tel développement (à l'ordre 2) de la solution d'une équation différentielle stochastique a été introduit par P. Malliavin dans [12] et [13], où l'on voit apparaître dans le terme d'ordre 2 les commutateurs des champs de vecteurs définissant l'équation multipliés par des intégrales de P. Lévy. On veut ici généraliser ce type de paramétrix 
trajectorielle et montrer que ce formalisme contient et étend les représentations explicites des solutions d'équations stochastiques obtenues par B. Gaveau [8], Y. Yamato [18], M. Fliess-D. Normand-Cyrot [7], Krener-Lobry [19] et H. Kunita [11]. Cette représentation explicite donne la solution comme une fonctionnelle des intégrales itérées du mouvement brownien.

Nous montrons que cette représentation n'est rien d'autre qu'un développement de Taylor dans un bon système de cartes. Cette approche permet d'étendre ces résultats au cas où l'algèbre de Lie engendrée par les champs de vecteurs qui définissent l'équation n'est pas nilpotente mais seulement de dimension finie. Elle permet aussi, dans un autre contexte, de donner une représentation explicite et universelle du mouvement brownien sur une variété riemannienne en fonction des dérivées covariantes du tenseur de courbure et des intégrales itérées du mouvement brownien euclidien.

Passons au plan détaillé de ce qui suit:

La solution d'une équation differentielle stochastique (sour forme Stratonovitch) à coefficients infiniment differentiables se développe, en temps petit, en fonction des intégrales de Stratonovitch itérées du temps et du mouvement brownien qui définit l'équation. Ces intégrales itérés se réduisent, évidemment, aux monomes $t^{m} / m$ ! dans le cas d'une équation différentielle ordinaire.

Dans [1] Azencott obtient une formule de Taylor stochastique précise, à savoir une majoration du reste en probabilité lorsque $t$ tend vers zéro (rappelée ici au $\S 2$ théorème 6). Pour les applications aux développements limités de fonctionnelles de la solution voir Azencott [1] et [2].

On commence ici, dans le $\S 1$, par étudier la convergence de la série de Taylor stochastique de la solution d'une équation stochastique à coefficients analytiques. On montre (théorème 9) que la solution d'une équation stochastique à coefficients analytiques est la somme de sa série de Taylor stochastique, ceci avant un temps d'arrêt presque surement strictement positif. La preuve de ce résultat est fondée sur un théorème de dépendance analytique des paramètres et de la condition initiale de la solution d'une équation stochastique à coefficients analytiques (théorème 5). Ce type de convergence avant un temps d'arrêt est clairement le mieux que l'on puisse esperer en général. Cependant une étude directe des séries de Taylor stochastiques menée au $§ 1$ a) permet d'obtenir des critères concrets de convergence de ces séries sur un intervalle de temps déterministe et même pour tout $t$ (théorème 2 , corollaire 1 et proposition 3 ). Ces critères permettent de voir la famille infinie des intégrales itérées comme une diffusion à valeurs dans un espace de Banach (théorème 3 ) et donc, d'une certaine façon, comme une "diffusion universelle», puisqu'en temps petit la solution d'une équation à coefficients analytiques est une fonctionnelle régulière universelle des champs de vecteurs qui définissent l'équation et de cette diffusion de dimension infinie.

On montre ensuite que les résultats classiques de Doss [6] et Sussmann [17] qui lient le flot d'une équation stochastique et celui d'une équation différentielle ordinaire (aléatoire et dépendant du temps) lorsque les champs de vecteurs $X_{i}$ qui définissent l'équation commutent ou ceux de Yamato [18], Kunita [11], Fliess-Norman Cyrot [7], Krener-Lobry [19] dans le cas où les $X_{i}$ engendrent une algèbre de Lie nilpotente, sont des conséquences directes du développement en série de Taylor stochastique. 
Il est non seulement possible de retrouver ces résultats connus mais aussi de montrer que cette technique s'étend au cas où les $X_{i}$ engendrent une algèbre de Lie quelconque de dimension finie (théorème 19 et corollaires 2 et 3) même si les champs $X_{i}$ ne sont pas complets (théorème 20).

On montre d'abord sous des hypothèses générales ( $\$ 3$ théorèmes 10 et 11) que le flot défini par l'équation stochastique est le flot au temps $t=1$ d'un champ de vecteurs $X(t, w)$ aléatoire et dépendant du temps, que l'on peut décrire en temps petit au moyen de la formule de Taylor stochastique. Le résultat ainsi obtenu ne contient pas encore les résultas connus dans les cas abélien et nilpotent car la description de ce champ de vecteur $X(t, w)$ n'est pas suffisamment intrinsèque.

Pour préciser ce résultat, sous l'hypothèse supplémentaire de dimension finie de l'algèbre de Lie engendrée par les champs $X_{i}$, et obtenir le théorème 19 on commence par étudier le cas d'une équation invariante sur un groupe de Lie et donner une expression totalement explicite, en carte exponentielle, du développement de Taylor stochastique de la solution (théorème 12) ce qui constitue le résultat central de cet article.

Dans le cas d'un groupe nilpotent $(\$ 4 b)$ on vérifie (théorème 17) que cette méthode donne la même expression que celle obtenue en appliquant l'idée de Kunita (11) qui consiste à utiliser l'approximation des intégrales multiplicatives (Ibéro [9]) et la formule de Campbell-Hausdorff. Pour cela on fait le lien entre intégrales stochastiques multiples au sens de Meyer [14] qui apparaissent ici naturellement (comme dans Kunita [11]) et la famille des intégrales itérées. La preuve de l'identité de ces deux développements est a priori inutile mais elle est donnée car elle met en jeu une interaction "miraculeuse» entre des formules purement algébriques sur la série de Campbell-Hausdorff et des identités probabilistes entre intégrales itérées d'Ito et de Stratonovitch (reste à savoir si ces identités sont vraiment probabilistes; pour les aspects algèbriques et formels voir Fliess et Normand-Cyrot [7]).

Enfin le (c) du $\S 4$ fait le lien entre ces formules et la représentation de la solution obtenue dans la cas résoluble par Kunita et on explicite l'exemple du groupe affine sur $\mathbf{R}$.

L'application des résultats du $\S 4$ valables sur un groupe au cas d'une équation sur une variété et l'obtention des théorèmes 19,20 et des corollaires 2 et 3 qui étendent les résultats de $[7,11,15]$ est alors immédiate par la réciproque $\mathrm{du}$ second théorème de Lie pour un résultat en temps petit et par le theorème de Palais [15] pour un résultat global (sous l'hypothèse de complétude des champs $X_{i}$ ).

La question reste ouverte de savoir si dans le cadre général du $\S 3$, ie sans l'hypothèse de dimension finie de l'algèbre de Lie engendrée par les champs $X_{i}$, le champ de vecteurs $X(t, w)$ admet pour développement de Taylor stochastique celui que le corollaire 2 lui assignerait, ou encore l'hypothèse de dimension finie est elle nécessaire dans le corollaire 2?

Enfin on montre dans le $\$ 6$ que ces techniques peuvent s'appliquer dans une autre direction pour décrire, en temps petit, le mouvement brownien sur une variété riemannienne. Ainsi on montre (théorème 21) qu'en carte normale les coefficients du développement de Taylor stochastique du Brownien sont des 
polynomes universels des dérivées covariantes successives du tenseur de courbure; à l'ordre 2 le mouvement brownien sur la variété est euclidien, les corrections due à la courbure n'apparaissant qu'à l'ordre 3.

\section{Intégrales stochastiques itérées et séries de Taylor stochastiques}

Considérons un mouvement brownien $r$-dimensionnel $\left(B_{t}^{1} \ldots B_{t}^{r}\right)$. Notons, pour simplifier, $B_{t}^{0}=t$. Si $J=\left(j_{1} \ldots j_{m}\right) \in\{0 \ldots r\}^{m}$, nous noterons $B_{t}^{J}$ l'intégrale stochastique itérée de Stratonovich

$$
\int_{0<t_{1}<\ldots<t_{m}<t} d B_{t_{1}}^{j_{1}} \ldots \ldots d B_{t_{m}}^{j_{m}} .
$$

Considérons la famille infinie $Y_{t}$ de toutes ces intégrales itérées $Y_{t}$ $=\left(B_{t}^{J}\right)_{J \in\{0 \ldots r\} m}, m \in N^{*}$. Il s'agit d'un processus à valeurs dans l'espace $\mathscr{T}\left(\mathbb{R}^{r+1}\right)$ $=\bigoplus_{m=1}^{\infty}\left(\mathbb{R}^{r+1}\right)^{\otimes^{m}}$.

Pour $y \in \mathscr{T}\left(\mathbb{R}^{r+1}\right)$, notons $y^{(m)} 1$ s composante de $y$ sur $\left(\mathbb{R}^{r+1}\right)^{\otimes^{m}} \cdot y^{(m)}$ s'écrit à son tour $y^{(m)}=\left(y^{J}\right)_{J \in\{0 \ldots r\}^{m}}$ dans la base canonique de $\left(\mathbb{R}^{r+1}\right)^{\otimes^{m}}$. Ici, évidemment, $Y_{t}^{(m)}=\left(B_{t}^{J}\right)_{J \in\{0 \ldots r\}^{m}}$.

Définissons les séries de Taylor stochastiques.

Définition. Si $x \in \mathscr{T}\left(\mathbb{R}^{r+1}\right)$, on appellera série de Taylor stochastique définie par $x$ l'élément $y$ de $\mathscr{T}\left(\mathbb{R}^{r+1}\right)$ défini par $y^{J}=x^{J} B_{t}^{J} \forall J \in \bigcup_{1}^{\infty}\{0 \ldots r\}^{m}$.

Dans la suite, nous allons donner des critères pour la convergence des séries $\sum_{m=1}^{\infty} \sum_{|J|=m} y_{J}$ ou $\sum_{m=1}^{\infty} \sum_{\|J\|=m} y_{J}$, avec $\|J\|=|J|+\operatorname{card}\left\{i / j_{i}=0\right\}$.

\section{(a) Intégrales d'Ito itérées}

Pour cela, commençons par étudier les intégrales itérées au sens d'Ito (Riemann) $I_{t}^{J}=\int_{0<t_{1}<\ldots<t_{m}<t} \ldots B_{t_{1}}^{j_{1}} \ldots d B_{t_{m}}^{j_{m}}$. Soit $J=\left(j_{1} \ldots j_{m}\right) \in\{0 \ldots r\}^{m}$. Notons

$$
\begin{aligned}
& a_{1}=\inf \left(i, j_{i} \neq 0\right) \\
& b_{1}=\inf \left(i>a_{1}, j_{i}=0\right) .
\end{aligned}
$$

Puis $a_{n}=\inf \left(i>b_{n-1}, j_{i} \neq 0\right), b_{n}=\inf \left(i>a_{n}, j_{i}=0\right)$ pour les $n$ tels que ces infima soient bien définis.

Définissons alors $p_{1}^{(J)}=a_{1}-1$ si $a_{1}$ défini, $p_{1}=m$ sinon. Puis $p_{n}(J)=a_{n}-b_{n-1}$ pour les $n$ tels que $a_{n}$ et $b_{n-1}$ sont définis. Et $P_{n}(J)=m-b_{n-1}$ si $b_{n-1}$ est défini et $a_{n}$ ne l'est pas. $p_{i}(J)$ est alors la longueur du $i^{\text {eme }}$ bloc de zéros consécutifs dans $J$. Soit $k(J)$ le nombre total de tels blocs de zéros consécutifs dans $J$. On a alors l'égalité: 
Théorème 1. $\forall t \geqq 0 \quad E\left|I_{t}^{J}\right|^{2}=\frac{t^{\|J\|}}{a(J)}$ avec $a(J)=\|J\| ! \prod_{i=1}^{k(J)} \frac{p_{i} !^{2}}{\left(2 p_{i}\right) !}$. La preuve de ce théorème est renvoyée en appendice.

Remarque. On a une description précise de $a(J)$. Si l'on cherche seulement à encadrer $a(J)$, on peut écrire que: $|J| ! \leqq a(J) \leqq\|J\|$ ! ce qui est clair sur la forme de $a(J)$, mais aurait pu s'obtenir avec bien moins d'efforts. On peut donner un encadrement de $a(J)$ qui sera utile plus loin. On $a$ :

Lemme 1. $\left(\frac{1}{4}\right)_{i}^{k} p_{i} \mid\|J\| ! \leqq a(J) \leqq\left(\frac{1}{2}\right)_{i}^{k} p_{i}\|J\| !$

Preive. Pour tout $p \quad C_{2(p+1)}^{p+1}=2\left(1+\frac{p}{p+1}\right) C_{2 p}^{p}$. D'où $2^{p} \leqq C_{2 p}^{p} \leqq 4^{p}$ et donc $\left(\frac{1}{4}\right)^{\sum_{i}^{k} p_{i}} \leqq \prod_{i}^{k} p_{i} !^{2}\left(2 p_{i}\right) ! \leqq\left(\frac{1}{2}\right)^{\sum_{i}^{k} p_{i}}$ d'où le résultat.

Remarquons que l'exposant $\sum_{i}^{k} p_{i}$ est simplement le nombre total de zéros dans $J . \sum_{i}^{k} p_{i}=\|J\|-|J|$. En particulier, $\sum_{i}^{k} p_{i} \leqq|J|$.

\section{(b) Intégrales de Stratonovich itérées}

Il est possible d'utiliser les majorations précédentes pour majorer les intégrales de Stratonovich; en effet, on a de façon générale:

Proposition 1. Soient $X^{1} \ldots X^{m} m$ semi-martingales continues. Lintégrale de Stratonovich itérée $\int_{0<t_{1}<\ldots<t_{m}<t} \ldots X_{t_{1}}^{1} \circ \ldots \circ d X_{t_{m}}^{m}$ est une combinaison linéaire d'intégrales d'Ito itérées, précisément:

$$
\int_{0<t_{1}<\ldots<t_{m}<t} d x_{t_{1}}^{1} \circ \ldots \circ d x_{t_{m}}^{m}=\sum_{k=\left[\begin{array}{c}
m \\
2
\end{array}\right]}^{m} \frac{1}{2^{m-k}} \sum_{v \in A_{m}^{k}} I_{r}(v)
$$

où $A_{m}^{k}=\left\{v=\left(n_{1} \ldots n_{k}\right) \in\{1,2\}^{k} / \sum_{i}^{k} n_{i}=m\right\}$ et $I_{t}(v)$ est l'intégrale d'Ito(-Stieltjes) itérée définie par

$$
\begin{aligned}
I_{t}(v)=\int_{0<t_{1}<\ldots<t_{k}<t} d \ldots \int Y_{t_{1}}^{1} \ldots d Y_{t_{k}}^{k} \text { avec } & Y_{s}^{i}=X^{p_{j}} \text { si } n_{i}=1 \text { et } p_{j}=\sum_{i=1}^{j} n_{i} \\
& \text { et } \\
& Y_{s}^{i}=\left\langle X^{p_{j}-1^{c}}, X^{p_{j}^{c}}\right\rangle \text { si } n_{i}=2 .
\end{aligned}
$$


La preuve de cette proposition est une récurrence évidente à partir de la définition de l'intégrale de Stratonovich.

En particulier, ceci montre que:

$$
B_{t}^{J}=\sum_{k=m-m(J)}^{m} \frac{1}{2^{m-k}} \sum_{v \in A_{m}^{k}} c_{v} I_{t}^{J}(v)
$$

où $J(v)=\left(l_{1} \ldots l_{k}\right)$ et

$$
\begin{gathered}
\begin{cases}l_{s}=j_{p_{s}} & \text { si } n_{s}=1 \\
l_{s}=0 & \text { si } n_{s}=2\end{cases} \\
\text { pour } v=\left(n \ldots n_{t}\right) \text { et } p_{s}=\sum_{i}^{s} n_{i}, \text { et } \\
\begin{cases}c_{v}=1 & \text { si } j_{p_{s}-1}=j_{p_{s}} \neq 0 \forall s \\
c_{v}=0 & \text { sinon }\end{cases}
\end{gathered}
$$

et $m(J)$ est le nombre maximal de paires $\left(j_{i}, j_{i+1}\right)$ d'indices de $J$ tels que $j_{i}=j_{i+1}$ $\neq 0 . m-m(J)$ est supérieur ou égal à $\left[\frac{|J|}{2}\right]$. On a toujours $|J(v)|=k$ et $\|J(v)\|=\|J\|$.

Remarque. Cette expression des intégrales de Stratonovich comme combinaison linéaire d'intégrales d'Ito itérées d'ordre inférieur ou égal est inversible et montre aussi que toute intégrale d'Ito itérée est une combinaison linéaire d'intégrales de Stratonovich itérées d'ordre inférieur ou égal.

On peut alors majorer en norme $L^{2}$ les intégrales de Stratonovich itérées:

\section{Lemme 2.}

$$
E\left(\left|B_{t}^{J}\right|^{2}\right) \leqq b(J)^{2} t^{\|J\|}
$$

avec

$$
b(J)=\sum_{b=m-m(J)}^{m} \frac{1}{2^{m-k}} \sum_{v \in A_{m}^{k}} c_{v} \frac{1}{\sqrt{a(J(v))}} .
$$

Il suffit d'écrire $\left\|B_{t}^{J}\right\|_{L^{2}} \leqq \sum_{k=m}^{m} \frac{1}{2^{m-k}} \sum_{v \in A_{m}^{k}} c_{v}\left\|I_{t}^{J(v)}\right\|_{L^{2}}$ et d'utiliser le lemma 1.

L'expression des $a(J)$ permet de majorer les coefficients $b(J)$ :

Lemma 3. $b(J) \leqq\left(\frac{5}{2}\right)^{|J|} \frac{1}{\sqrt{\|J\| !}}$

Preuve. Pour tout $v \in A_{m}^{k}$, on a vu que $|J(v)|=k$ et $\|J(v)\|=\|J\|$ d'où $a(J(v))$ $\left.\geqq\left(\frac{1}{4}\right)\right)^{|J(v)|}\|J(v)\| !=\left(\frac{1}{4}\right)^{k}\|J\| !$ D'où

$$
b(J) \leqq \frac{1}{\sqrt{\|J\| !}} \sum_{k=m-m(J)}^{m}\left(\frac{1}{2^{m-k}}\left(\sum_{v \in A_{m}^{k}} c_{v}\right) 2^{k}\right) .
$$

Or Card $A_{m}^{k}=C_{m}^{m-k}$ d'où, en majorant tous les $C_{v}$ par 1 , on obtient:

$$
b(J) \leqq\left(\sum_{k=m-m(J)}^{m} C_{m}^{m-k} \frac{1}{2^{m-k}} 2^{k}\right) \frac{1}{\sqrt{\|J\| !}} .
$$


En minorant $m-m(J)$ par 0 , on $a: b(J) \leqq\left(\frac{1}{2}+2\right)^{m} \frac{1}{\sqrt{\|J\| !}}=\left(\frac{5}{2}\right)^{|J|} \frac{1}{\sqrt{\|J\| !}}$.

Remarque. Le coefficient $\frac{5}{2}$ n'est pas optimal, mais une majoration de $b(J)$ par $C \cdot \lambda^{|J|} \frac{1}{\sqrt{\|J\| !}}$ est naturelle avec $\lambda>1$. Par exemple, si $J=(1 \ldots 1)$ alors $B_{t}^{J}=\frac{B^{1 m}}{m !}$, d'où

$$
E\left(B_{t}^{J^{2}}\right)=\frac{2 m !}{m !^{3} 2^{m}} t^{2 m}
$$

et donc

$$
\frac{2}{3}\left(\frac{3}{3}\right)^{m} \frac{t^{2 m}}{m !} \leqq E\left(B_{t}^{J^{2}}\right) \leqq 2^{m} \frac{t^{2 m}}{m !}
$$

et donc

$$
\sqrt{\frac{2}{3}}\left(\sqrt{\frac{3}{2}}\right)^{|J|} \frac{1}{\sqrt{\|J\| !}} \leqq b(J) \leqq \sqrt{2^{|J|}} \frac{1}{\sqrt{\|J\| !}}
$$

(c) Convergence des séries de Taylor stochastiques

On arrive enfin aux théorèmes de convergence des séries de Taylor stochastiques:

Théorème 2. Soit $x \in \mathscr{T}\left(\mathbb{R}^{r+1}\right)$.

(1) Si $\sum_{m} \sum_{|J|=m}\left|x_{j}\right| b(J) \sqrt{t^{\|I\|}}<\infty$ (resp. $\left.\sum_{m}\left(\sum_{\|J\|=m}\left|x_{J}\right| b(J)\right) \sqrt{t^{m}}<\infty\right)$ alors la série $\sum_{m} \sum_{|J|=m}\left|x_{J} B_{t}^{J}\right|_{L^{2}}$ converge (resp. $\sum_{m} \sum_{\|J\|=m}\left|x_{J} B_{t}^{J}\right|_{L^{2}}$ converge.

(2) Si $\sum_{m} \sum_{|J|=m}\left|x_{J}\right|^{2} b(J)^{2} t^{\|J\|}<\infty$ (resp. $\sum_{m}\left(\sum_{\|J\|=m}\left|x_{J}\right|^{2} b(J)^{2}\right) t^{m}<\infty$ alors la série $\sum_{m} \sum_{|J|=m}\left|x_{J} B_{t}^{J}\right|\left(\right.$ resp. $\left.\sum_{m} \sum_{\|J\|=m}\left|x_{J} B_{t}^{J}\right|\right)$ converge ps uniformément sur $\left[0, t^{\prime}\right]$ pour tout $t^{\prime} \leqq \frac{t}{r+1}$.

Preuve. Le (1) est une conséquence évidente du lemme 2. Pour le (2), on a: si $t^{\prime}<\frac{t}{r+1}$ et $C=\frac{t^{\prime}}{t}(r+1)<1$; si $s<t^{\prime}$ :

$$
\begin{aligned}
P\left(\sum_{|J|=m}\left|x_{J} B_{s}^{J}\right| \geqq C^{m}\right) & \leqq \frac{1}{C^{2 m}} E\left(\left(\sum_{|J|=m}\left|x_{J} B_{s}^{J}\right|\right)^{2}\right) \\
& \leqq \frac{(r+1)^{m}}{C^{2 m}} \sum_{|J|=m}\left|x_{J}\right|^{2}\left|B_{s}^{J}\right|_{L^{2}}^{2} \\
& \leqq \sum_{|J|=m}\left|x_{J}\right|^{2} b(J)^{2} S^{\|J\|}\left(\frac{r+1}{C^{2}}\right)^{m} .
\end{aligned}
$$


Or $\left(\frac{s}{t}\right)^{\|J\|} \leqq\left(\frac{s}{t}\right)^{m}$ d'où $\frac{s^{\|J\|}}{t^{\|J\|}}\left(\frac{r+1}{C^{2}}\right)^{m} \leqq\left(\frac{(r+1) s}{C^{2} t}\right)<1$

D'où $s^{\|J\|}\left(\frac{r+1}{C^{2}}\right)^{m} \leqq t^{\|J\|}$ et: $P\left(\sum_{|J|=m}\left|x_{J} B_{\mathrm{s}}^{J}\right| \geqq C^{m}\right) \leqq \sum_{|J|=m}\left|x_{J}\right|^{2} b(J)^{2} t^{\|J\|}$.

La serie de droite est convergente. Le lemme de Borel-Cantelli donne le résultat. (Le calcul est analogue pour les séries $\sum_{\|J\|=m} x_{J} B_{t}^{J}$ ).

Remarque. Si la série de Taylor est déterministe, à savoir si $x_{J}$ est nul dès que l'un des $j_{i}$ est non nul, notons $x_{m}=x_{J}$ pour $J=(\underbrace{0 \ldots 0}_{m \text { fois }})$. Alors $\sum_{m} \sum_{|J|=m}\left|x_{J} B_{t}^{J}\right|_{L^{2}}=\sum_{m}|x|_{m} \frac{t^{m}}{m !}$. On a alors $b(J)=\frac{1}{\sqrt{a(J)}}=\frac{1}{\sqrt{m !^{2}}}=\frac{1}{m !}$ et $\|J\|=2 m$ pour un tel $J$ et la condition (1) s'écrit $\sum_{m}\left|x_{m}\right| \frac{t^{m}}{m !}<\infty$; elle est donc évidemment optimale.

Les majorations de $b(J)$ obtenues au lemme 3 permettent d'obtenir des critères concrets des séries de Taylor stochastiques.

Corollaire 1. Soit $x \in \mathscr{T}\left(\mathbb{R}^{r+1}\right)$ tel que:

$$
\exists K>0 \text { et } \exists \alpha \in \mathbb{R} \quad\left|x_{J}\right| \leqq K^{|J|}\|J\| !^{\alpha} .
$$

(1) Si $\alpha<1 / 2$.

La série $\sum_{m} \sum_{|J|=m}\left|x_{J} B_{t}^{J}\right|_{L^{2}}$ (respectivement $\left.\sum_{m} \sum_{\|J\|=m}\left|x_{J} B_{t}^{J}\right|_{L^{2}}\right)$ converge pour tout $t$.

La série $\sum_{m} \sum_{|J|=m}\left|x_{J} B_{t}^{J}\right|\left(\right.$ respectivement $\left.\sum_{m} \sum_{\|J\|=m}\left|x_{J} B_{t}^{J}\right|\right)$ converge presque surement uniformément sur tout intervalle $[0, T]$.

(2) Si $\alpha=1 / 2$.

Il existe des réels $0<t_{0}<t_{1}$ tels que: La série $\sum_{m} \sum_{|J|=m}\left|x_{J} B_{t}^{J}\right|_{L^{2}}$ (respectivement $\left.\sum_{m} \sum_{\|J\|=m}\left|x_{J} B_{t}^{J}\right|_{L^{2}}\right)$ converge pour $t<t_{0}$.

La série $\sum_{m} \sum_{|J|=m}\left|x_{J} B_{t}^{J}\right|$ (respectivement $\left.\sum_{m} \sum_{\|J\|=m}\left|x_{J} B_{t}^{J}\right|_{L^{2}}\right)$ converge presque surement uniformément sur $\left[0, t_{1}\right]$.

Preuve. On se limitera au calcul pour les séries $\sum_{m} \sum_{|J|=m} x_{J} B_{t}^{J}$. On a

$$
\begin{aligned}
\sum_{|J|=m}\left|x_{J}\right| b(J) \sqrt{t^{\| J} \mid} & \leqq \sum_{|J|=m}\left(\frac{5}{2} K(\sqrt{t} \vee t)\right)^{m} 2 m !^{\alpha-1 / 2} \\
& \leqq\left[(r+1) \frac{5}{2} K(\sqrt{t} \vee t)\right]^{m} 2 m !^{\alpha-1 / 2}
\end{aligned}
$$

et

$$
\sum_{|J|=m}\left|x_{J}\right|^{2} b(J)^{2} t^{\|\cdot I\|} \leqq\left((r+1) \frac{25}{4} K^{2} t \vee t^{2}\right)^{m} 2 m !^{2 \alpha-1}
$$


Si $\alpha<1 / 2$, ces séries convergent pour tout $t$ d'où le 1) du corollaire. Si $\alpha=1 / 2$, la première de ces séries converge pour $(\sqrt{t} \vee t)<\frac{2}{5(r+1) K}=\lambda$ donc pour $t$ $<\inf \left(\lambda, \lambda^{2}\right)=t_{0}$.

La seconde converge pour $t<\inf \left(\mu, \mu^{2}\right)$ avec $\mu=\frac{2}{5 \sqrt{r+1} K}$, ce qui démontre le 2) du corollaire avec $t_{1}=\frac{1}{r+1} \inf \left(\mu, \mu^{2}\right)$.

Remarque. Les rayons de convergence $t_{0}$ et $t_{1}$ ne sont pas optimaux. Par contre, l'exposant critique $\alpha=1 / 2$ l'est.

Pour la convergence $\mathrm{p} \cdot \mathrm{s}$, considérons de nouveau le cas extrême d'une série de Taylor déterministe: $x_{J}=0$ si l'un des $j_{i}$ est non nul, et notons $x_{J}=x_{m}$ pour $J=(\underbrace{0 \ldots 0}_{m \text { fois }})$.

L'hypothèse $\left|x_{J}\right| \leqq K^{|J|}\|J\| !^{\alpha}$ s'écrit ici $\left|x_{m}\right| \leqq K^{m}(2 m) !^{\alpha}$. La formule de Stirling montre que la série de Taylor $\sum_{m} \sum_{|J|=m}\left|x_{J} B_{t}^{J}\right|=\sum_{m} x_{m} \frac{t^{m}}{m !}$ converge pour tout $t$ si $\alpha<1 / 2$ et pour tout $t \in\left[0, \frac{1}{2 K}[\right.$ si $\alpha=1 / 2$, mais diverge pour tout $t$ si $\alpha>1 / 2$ et $\left|x_{m}\right| \geqq K^{m}(2 m) !^{\alpha}$.

Considérons un cas simple non déterministe en posant:

$$
\begin{aligned}
& x_{J}=K^{m}(m !)^{\alpha}=K^{|J|}\|J\| !^{\alpha} \quad \text { si } J=(\underbrace{1 \ldots 1}_{m \text { fois }}) \\
& x_{J}=0 \text { sinon. }
\end{aligned}
$$

Alors, de même la formule de Stirling montre que la série de Taylor stochastique

$$
\sum_{m} \sum_{|J|=m}\left|x_{J} B_{t}^{J}\right|_{L^{2}}=\sum_{m} K^{m}(m !)^{\alpha}\left|\frac{B_{t}^{m}}{m !}\right|_{L^{2}}=\sum_{m} K^{m} m !^{\alpha-1} \sqrt{\frac{2 m !}{m ! 2^{m}}} t^{m}
$$

converge pour tout $t$ si $\alpha<1 / 2$, converge pour tout $t<1 / 2 K$ si $\alpha=1 / 2$ et diverge si $\alpha>1 / 2$ pour tout $t$.

Par contre, dans ce cas, la convergence $\mathrm{p} \cdot \mathrm{s}$ a lieu pour tout $t$ pour $\alpha<1$ mais pour $\alpha=1$, la série $\sum_{m}\left|\sum_{|J|=m} x_{J} B_{t}^{J}\right|$ ne converge $p \cdot s$ sur aucun intervalle déterministe $[0, T]$.

Pourtant, cette série converge dans un sens plus faible, elle a un rayon de convergence aléatoire.

Plus précisément, si $T$ est le temps d'arrêt $T=\inf \left(t,\left|B_{t}^{1}\right|=1 / K\right)$ alors $\mathrm{p} \cdot \mathrm{s}$ sur l'intervalle stochastique $\left[0, T\left[\right.\right.$ la série $\sum_{m} \sum_{|J|=m}\left|x_{J} B_{t}^{J}\right|$ converge. Nous allons, dans les paragraphes suivants, nous intéresser à ce type de convergence avant un temps d'arrêt. 
(d) La famille des intégrales itérées comme diffusion sur un espace de Banach

Soit la famille infinie $Y_{t}$ des intégrales stochastiques itérées de Stratonovich; nous allons montrer ici qu'il s'agit bien d'un processus de diffusion à valeurs dans un espace de Banach (et pas seulement dans le «gros» espace $\mathscr{T}\left(\mathbb{R}^{r+1}\right)$ ).

Soit $H$ le sous-espace de $\mathscr{T}\left(\mathbb{R}^{r+1}\right)$ donné par:

$$
H=\left\{x \in \mathscr{T}\left(\mathbb{R}^{r+1}\right) / \sum_{m} \sum_{|J|=m}\left|x^{J}\right|<\infty\right\}
$$

$H$ muni de la norme $\|x\|=\sum_{m} \sum_{|J|=m}\left|x^{J}\right|$ est évidemment un espace de Banach. On a alors:

Theoreme 3. (1) Presque surement, pour tout $t Y_{t} \in H$.

(2) $\left(Y_{t}\right)$ est la solution sur $H$ de l'équation stochastique

$$
d Y_{t}=\sum_{j=0}^{r} Q_{j}\left(Y_{t}\right) \circ d B_{t}^{j}
$$

où les champs $Q_{j}$ sont donnés par:

$$
\text { Si } J=\left(j_{1} \ldots j_{m}\right) \quad \text { si } Q_{j}^{J}(y)= \begin{cases}0 & \text { si } j \neq j_{m} \\ y^{j_{1} \ldots j_{m-1}} & j=j_{m}\end{cases}
$$

Preuve. (1) $Y_{t}$ est la série de Taylor stochastique donnée par $x_{J}=1$ pour tout $J$. Les critères du corollaire assurent que $\sum_{m} \sum_{|J|=m}\left|x_{J} B_{t}^{J}\right|$ converge $p \cdot s$ uniformé ment sur $[0, T]$ pour tout $T$ d'où le (1).

(2) On vérifie trivialement que les $Q_{j}$ définissent des champs de vecteurs sur $H$. L'équation (2) est la définition des intégrales itérées.

Ainsi, la famille $\left(Y_{t}\right)$ est une diffusion de dimension infinie. Les résultats des paragraphes suivants vont montrer dans quelle mesure il s'agit de la «diffusion universelle», à savoir: on va exprimer toute diffusion analytique comme une fonctionnelle régulière de cette diffusion et approximer toute diffusion à coefficients $C^{\infty}$ par une telle fonctionnelle.

\section{§ 2. Formule de Taylor stochastique}

(a) E.D.S. à coefficients $C^{\infty}$

Soit $U$ un ouvert de $\mathbb{R}^{m}, E$ un ouvert de $\mathbb{R}^{p}$ et $\sigma_{0}, \ldots, \sigma_{r} r+1$ applications $C^{\infty}$ de $U \times E$ à valeurs dans $\mathbb{R}^{n}$. Considérons l'équation stochastique de Stratonovich sur $U$ :

$$
\begin{aligned}
d X_{t} & =\sum_{i=0}^{r} \sigma_{i}\left(\varepsilon, X_{t}\right) \circ d B_{t}^{i} \\
X_{0} & =x
\end{aligned}
$$


où l'on a noté, pour simplifier, $B_{t}^{0}=t$. Pour un borelien $A$ de $U$, notons $X_{t}(\varepsilon, x)$ la solution de (1) et $T_{A}^{\varepsilon, x}$ le temps de première sortie de $X_{t}(\varepsilon, x)$ hors de $A$

$$
T_{A}^{\varepsilon, x}=\inf \left(t, X_{t}(\varepsilon, x) \notin A\right) .
$$

On a alors le résultat classique suivant:

Théorème 4. (1) Si $U=\mathbb{R}^{m}, E=\mathbb{R}^{p}$ et si les champs $\sigma_{i}$ sont bornés à dérivées bornées, alors presque surement, pour tout $t, X_{t}(\cdot, \cdot)$ est $C^{\infty}$ en $(\varepsilon, x)$ sur $\mathbb{R}^{m} \times \mathbb{R}^{p}$.

(2) Dans le cas général où $\sigma_{i}$ est $C^{\infty}$ sur $U \times E$, soit $K$ un compact de $U$ et $V$ (respectivement $F$ ) un ouvert borné non vide de $\mathbb{R}^{m}$ (respectivement $\mathbb{R}^{p}$ ) tel que $\bar{V} \subset K^{\circ}$. Alors presque surement pour $t<T=\inf _{\substack{i \in F \\ x \in V}} T_{K}^{\varepsilon, x}, X_{t}(\cdot, \cdot)$ est $C^{\infty}$ sur $F \times V$.

De plus T est un temps d'arrêt presque surement strictement positif.

Preuve. (1) Ce résultat est classique (cf. Bismut [4]) si les $\sigma_{i}$ ne dépendent pas de $\varepsilon$. Il est trivial de se ramener à ce cas. Il suffit de poser:

$$
\begin{aligned}
\tilde{\sigma}_{i}(\varepsilon, x) & =\left(0, \sigma_{i}(\varepsilon, x)\right) \\
\text { et } Y_{t}(\varepsilon, x) & =\left(\varepsilon, X_{t}(\varepsilon, x)\right) .
\end{aligned}
$$

alors $Y_{t}$ est solution sur $\mathbb{R}^{m} \times \mathbb{R}^{p}$ de l'équation:

$$
\begin{aligned}
Y_{0} & =(\varepsilon, x) \\
d Y_{t} & =\sum_{i=0}^{r} \tilde{\sigma}_{i}\left(Y_{t}\right) \circ d B_{t}^{i} .
\end{aligned}
$$

Ainsi, presque surement, pour tout $t Y_{t}$ est $C^{\infty}$ sur $\mathbb{R}^{m} \times \mathbb{R}^{p}$ et donc $X_{t}$ l'est.

(2) Si l'on remplace les champs $\sigma_{i}$ par des champs $\tilde{\sigma}_{i}$ bornés à dérivées bornées tels que $\tilde{\sigma}_{\left.i\right|_{K}}=\sigma_{\left.i\right|_{K}}$ et si $\tilde{X}_{t}$ désigne la solution de l'équation associée aux $\tilde{\sigma}_{i}$, alors $p \cdot s$ pour tout $t \tilde{X}_{t}$ est $C^{\infty}$ en $(\varepsilon, x)$ et pour $t<T X_{t}(\varepsilon, x)$ coïncident pour $(\varepsilon, x) \in F \times V$. D'où le résultat.

Le fait que $T$ soit un temps d'arrêt est évident. En effet, $T=\inf _{(\varepsilon, x) \in F \times V} T_{K}^{s, x}$ $=\inf _{\varepsilon, x \in D} T_{K}^{\varepsilon, x}$ si $D$ est un dénombrable dense de $F \times V$, donc $T$ est la borne inférieure d'un ensemble dénombrable de temps d'arrêt. Vérifions que presque surement $T$ est strictement positif. On a:

$$
T=\inf _{F \times V} T_{K}^{\varepsilon, x} \geqq \inf _{F \times V} T_{K}^{\varepsilon, x} \geqq \inf _{F \times V} T_{K}^{\varepsilon, x} .
$$

Or l'application $(\varepsilon, \chi) \rightarrow T_{K}^{\varepsilon, x}$ est s.c.i., sa borne inférieure est donc atteinte sur le compact $\overline{F \times V}$, elle est donc strictement positive.

Remarque. Si l'on s'intéresse à la seule régularité en fonction du paramètre $\varepsilon$, à $x_{0}$ fixé, on peut remplacer $T$ par $\inf _{\varepsilon \in F} T_{K}^{\varepsilon, x_{0}}$ qui, de même, est un temps d'arrêt strictement positif. 


\section{(b) E.D.S. à coefficients analytiques}

Supposons ici que les champs $\sigma_{i}$ soient analytiques réels au voisinage de $\left(\varepsilon_{0}, x_{0}\right) \in E \times U$; on va montrer la dépendance analytique du paramètre $\varepsilon$ et de la condition initiale $x$ pour la solution $X_{t}(\varepsilon, x)$ de (1).

Plus précisément, soient $P=P_{\varepsilon_{0}} \times P_{x_{0}}$ un pavé ouvert de $\mathbb{R}^{m} \times \mathbb{R}^{p}$, de centre $\left(\varepsilon_{0}, x_{0}\right)$ (où $P_{\varepsilon_{0}}$ (resp. $P_{x_{0}}$ ) est un pavé ouvert de centre $\varepsilon_{0}$ (resp. $\left.x_{0}\right)$ ) tel que $\bar{P}$ soit inclus dans l'intersection des domaines de convergence des séries des Taylor en $\left(\varepsilon_{0}, x_{0}\right)$ des $\sigma_{i}$. On a:

Théorème 5. Il existe un temps d'arrêt $T p \cdot s$ strictement positif, tel que $p \cdot s$ sur $t<T X_{t}(\cdot, \cdot)$ est analytique sur $P$.

Preuve. Par la réduction pratiquée pour le (1) du théorème précédent, on peut supposer que les champs $\sigma_{i}$ ne dépendent pas du paramètre $\varepsilon$. On considère donc l'équation sur $U$

$$
\begin{aligned}
d X_{t} & =\sum_{i=0}^{r} \sigma\left(X_{t}\right) d B_{t}^{i} \\
X_{0} & =x
\end{aligned}
$$

et l'on veut montrer l'existence d'un temps d'arrêt $T p \cdot s>0$ tel que sur $t<T X_{t}(\cdot)$ soit analytique sur $P_{1}$ (un pavé de centre $x_{0}$ et tel que $\bar{P}_{1}$ soit inclus dans l'intersection $V$ des domaines de convergence des séries de Taylor en $\left(\varepsilon_{0}, x_{0}\right)$ des $\left.\sigma_{i}\right)$.

On va, pour cela, complexifier l'équation (1). Précisons d'abord les notations:

Soit $\phi$ la bijection de $\mathbb{R}^{2 n}$ sur $\mathbb{C}^{n}$ suivante

$$
\phi\left(x_{1}, \ldots x_{2 n}\right)=\left(x_{1}+i x_{n+1}, \ldots, x_{n}+i x_{2 n}\right) .
$$

Si $W$ est un ouvert de $\mathbb{R}^{2 n}$, et $f$ une application $C^{1}$ de $W$ dans $\mathbb{R}^{n}$, alors $\hat{f}=\phi \circ f \circ \phi^{-1}$ définit une application sur l'ouvert $\phi(W)$ de $\mathbb{C}^{n}$ qui est analytique sur $\phi(W)$ si les conditions de Cauchy-Riemann sont satisfaites, à savoir si la matrice jacobienne de $f$ appartient à la sous-algèbre $M$ de $M_{2 n}(\mathbb{R})$ des matrices de la forme $\left(\frac{A}{B} \mid \frac{-B}{A}\right)$ où $A, B \in \mathscr{M}_{n}(\mathbb{R})$.

Démontrons maintenant le théorème:

Soit $P_{2}$ un pavé ouvert tel que $P_{1} \subset P_{2} \subset \bar{P}_{2} \subset V$. Soient alors $Q_{i}$ les polydisques ouverts de $\mathbb{C}^{n}$ tels que $Q_{i} \cap \mathbb{R}^{n}=P_{i}$.

Les séries de Taylor des $\sigma_{i}$ en $x_{0}$ définissent des applications analytiques $\tau_{0} \ldots \tau_{r}$ sur $Q_{2}$ telles que $\tau_{i_{\mid p_{2}}}=\sigma_{i}$. Sur l'ouvert de $\mathbb{R}^{2 n} \phi^{-1}\left(Q_{2}\right)$ ces applications $\tau_{i}$ définissent donc des applications $\eta_{i}=\phi^{-1} \circ \tau_{i} \circ \phi$ de classe $C^{\infty}$ dont les matrices jacobiennes appartiennent à $M$.

Prolongeons ces applications $\eta_{i}$ en des applications $\tilde{\eta}_{i}$ de classe $C^{\infty}$ sur $\mathbb{R}^{n}$ bornées à dérivées bornées et considérons l'équation sur $\mathbb{R}^{2 n}$ associée aux $\tilde{\eta}_{i}$ :

$$
\begin{aligned}
Y_{0}(y) & =y \in \mathbb{R}^{2 n} \\
d Y_{t}(y) & =\sum_{i=0}^{r} \tilde{\eta}_{i}\left(Y_{t}(y)\right) \circ d B_{t}^{i} .
\end{aligned}
$$


Par les résultats précédents, on sait que $Y_{t}(\cdot)$ est de classe $C^{\infty}$ sur $\mathbb{R}^{2 n}$. De plus, si l'on note $Z_{t}(y)$ la différentielle de $Y_{t}(\cdot)$ en $y$, on a l'équation aux variations dans $M_{2 n}(\mathbb{R})$ :

$$
\begin{aligned}
Z_{0}(y) & =I \\
d Z_{t}(y) & =\sum_{i=0}^{r} d \tilde{\eta}_{i}\left(Y_{t}(y)\right) \cdot Z_{t}(y) \circ d B_{t}^{i}
\end{aligned}
$$

Considérons $T=\inf _{y \in \phi^{-1}\left(Q_{2}\right)} T_{\phi^{-1}\left(Q_{2}\right)}^{y}$, alors, par un raisonnement déjà fait, $T$ est un temps d'arret $\mathrm{p} \cdot \mathrm{s}$ strictement positif. On va montrer que $\forall y \in \phi^{-1}\left(Q_{1}\right)$ $Z_{t \wedge T}(y) \in M$ et donc que, si $t<T, Y_{t}(\cdot)$ vérifie les conditions de Cauchy-Riemann $\operatorname{sur} \phi^{-1}\left(Q_{1}\right)$.

Posons $Z_{t}^{T}(y)=Z_{t \wedge T}(y), Z^{T}$ est l'unique solution de

$$
\begin{aligned}
Z_{0}^{T}(y) & =I \\
d Z_{t}^{T}(y) & =\sum_{i=0}^{r} A_{t}^{i} \cdot Z_{t}^{T}(y) 1_{t \leqq T} \circ d B_{t}^{i},
\end{aligned}
$$

avec $A_{t}^{i}=d \tilde{\eta}_{i}\left(Y_{t \wedge T}(y)\right)=d \eta_{i}\left(Y_{t \wedge T}(y)\right) \in M$, puisque $\eta_{i}$ vérifie les conditions de Cauchy-Riemann sur $\phi^{-1}\left(Q_{2}\right)$.

Or $M$ étant une sous-algèbre, l'équation $(*)$ a une solution unique dans $M$. D'où $\forall y \in \phi^{-1}\left(Q_{1}\right) Z_{t \wedge T}(y) \in M$.

Ainsi, si $t<T, Y_{t}(\cdot)$ définit une application de classe $C^{\infty}$ sur $\phi^{-1}\left(Q_{1}\right)$ dont la différentielle $Z_{t}(y)$ vérifie les conditions de Cauchy-Riemann. Donc $\hat{Y}_{t}(\cdot)$ $=\phi \circ Y_{t} \circ \phi^{-1}$ définie sur l'ouvert $Q_{1}$ de $\mathbb{C}^{n}$ est analytique si $t<T$.

De plus, par le fait que si $x \in P_{1} \quad \tilde{\eta}_{i}^{n+k}(x, 0)=\eta_{i}^{n+k}(x, 0)=\operatorname{Im}\left(\tau_{i}^{k}(x)\right)=0$ on vérifie que $Y_{i}^{n+k}(x, 0)=0$, donc que $Y_{i}(x, 0) \in \mathbb{R}^{n} \times\{0\}$ et donc $Y_{t \wedge T}(x, 0) \in \phi^{-1}\left(Q_{2}\right) \cap\left(\mathbb{R}^{n} \times\{0\}\right)=P_{2} \times\{0\}$.

Ainsi,

$$
\tilde{\eta}_{i}\left(Y_{t \wedge T}(x, 0)\right)=\eta_{i}\left(Y_{t \wedge T}(x, 0)=\sigma_{i}\left(Y_{t \wedge T}^{1}, \ldots, Y_{t \wedge T}^{n}(x, 0)\right)\right.
$$

et $\left(Y_{t \wedge T}^{1}(x, 0) \ldots Y_{t \wedge T}^{n}(x, 0)\right)$ est solution de l'equation stochastique vérifiée par $X_{t \wedge T}(x)$ sur $\mathbb{R}^{n}$ et l'on obtient que $\hat{Y}_{t \wedge T}(\cdot)$ coïncide avec $X_{t \wedge T}(\cdot)$ sur $P_{1}$.

Ce qui montre que, si $t<T, X_{t}(\cdot)$ est analytique sur $P_{1}$. C.Q.F.D.

(c) Formule de Taylor stochastique

Considérons de nouveau l'équation

$$
\begin{aligned}
d X_{t} & =\sum_{i=0}^{r} \sigma_{i}\left(X_{t}\right) \circ d B_{t}^{i} \\
X_{0} & =x_{0}
\end{aligned}
$$


où les champs de vecteurs $\sigma_{i}$ sont $C^{\infty}$ sur un ouvert $U$.

Ici, on va fixer la condition initiale $x_{0} \in U$ et étudier la dépendance en fonction de $t$ en temps petit. Si l'on avait affaire à une équation différentielle ordinaire, on pourrait affirmer qu'en temps petit $X_{t}\left(x_{0}\right)$ est $C^{\infty}$ en $t$ et écrire une formule de Taylor. Ici, un tel résultat est évidemment absurde; on va pourtant introduire une formule de Taylor stochastique due à Azencott [1] où les intégrales itérées

$$
B_{t}^{J}=\int_{0<t_{1}<\ldots<t_{m}<t} d B_{t_{1}}^{j_{1}} \ldots \circ d B_{t_{m}}^{j_{m}} .
$$

généralisent les monomes $\frac{t^{m}}{m !}=\int_{0<t_{1}<\ldots<t_{m}<t} d t_{1} \ldots d t_{m}$.

Notons $\sum_{v \in \mathbb{N}^{m}} a_{v}^{i}\left(x-x_{0}\right)^{v}$ la série de Taylor formelle de $\sigma_{i}$ en $x_{0}$ avec la convention usuelle de notation $x^{\nu}=x_{1}^{v_{1}} \ldots x_{m}^{v_{m}}$, si $v=\left(v_{1} \ldots v_{m}\right) \in \mathbb{N}^{m}$ et $x=\left(x_{1}, \ldots, x_{m}\right) \in \mathbb{R}^{m}$.

Si $g_{1}, \ldots, g_{n}$ désignent $n$ éléments de $\mathbb{R}^{m}$, soit le polynome à coefficients vectoriels $g_{1} x+\ldots+g_{n} x^{n}$ pour $x \in \mathbb{R}$ et soit alors $C_{i}^{k}\left(g_{1}, \ldots, g_{n}\right)$ le coefficient de $x^{k}$ dans la série formelle $\sum_{v \in \mathbb{N}^{m}} a_{v}^{i}\left(g_{1} x+\ldots+g_{n} x^{n}\right)^{v}$ obtenue par composition de série formelle de Taylor de $\sigma_{i}$ en $x_{0}$ et de ce polynome sans terme constant.

Nous pouvons alors introduire le système stochastique donné par:

$$
\mathscr{S}_{n}\left\{\begin{array}{l}
d g_{1}=\sum_{i=1}^{r} \sigma_{i}\left(x_{0}\right) \circ d B_{t}^{i} \\
d g_{2}=\sum_{i=1}^{r} C_{i}^{1}\left(g_{1}(t)\right) \circ d B_{t}^{i}+\sigma_{0}\left(x_{0}\right) \circ d B_{t}^{0} \\
d g_{n}=\sum_{i=0}^{r} C_{i}^{n-1}\left(g_{1} \ldots g_{n-1}\right) \circ d B_{t}^{i}+C_{0}^{n-2}\left(g_{1} \ldots g_{n-2}\right) \circ d B_{t}^{0}
\end{array}\right.
$$

avec les conditions initiales: $g_{i}(0)=0$.

La proposition suivante décrit les $g_{k}$ :

Proposition 2. (1) Pour tout $n \in \mathbb{N}^{*}$ le système stochastique $\mathscr{S}_{n}$ définit une diffusion $\left(g_{1} \ldots g_{n}\right)$ sur $\left(\mathbb{R}^{m}\right)^{n}$ à temps de vie infini. Chaque $g_{k}$ se calcule à partir d'intégrales itérées de Stratonovich précisément, si $g_{k}^{j}$ désigne la jème coordonnée de $g_{k}$; on $a$ :

$$
g_{k}^{j}(t)=\sum_{\|J\|=k} P_{J}^{j}\left(a_{v}^{i}\right) B_{t}^{J}
$$

où les $P_{J}^{i}$ sont des polynomes universels, à coefficients rationnels positifs, pris sur les coefficients de Taylor $a_{v}^{i}$ des champs $\sigma_{i}$.

(2) On a la propriété de scaling suivante:

$$
\begin{aligned}
& \text { Pour tout } t \geqq 0 \text { les processus } s \rightarrow\left(g_{1}(s t), \ldots, g_{n}(s t)\right) \\
& \text { et } s \rightarrow\left(t^{1 / 2} g(s), \ldots, t^{n / 2} g_{n}(s)\right)
\end{aligned}
$$

ont même loi. 
Remarque. On vérifie ainsi que les $g_{k}$ définissent une série de Taylor stochastique au sens précédent. On l'appellera série de Taylor stochastique associée à l'équation (1).

Preuve. (1) Il suffit de constater que $\mathscr{S}_{n}$ se résoud par quadratures stochastiques successives pour montrer qu'il définit une diffusion à temps de vie infini. Démontrons la deuxième assertion par récurrence en constatant qu'elle est claire pour $g_{1}$ car $g_{1}(t)=\sum_{i=1}^{r} \sigma_{i}\left(x_{0}\right) B_{t}^{i}$. Supposons que les coordonnées de $\left(g_{1}(t), \ldots, g_{n-1}(t)\right)$ soient des combinaisons linéaires d'intégrales stochastiques itérées; alors, puisque la $j^{\text {jeme }}$ coordonnée de $C_{i}^{k}\left(g_{1} \ldots g_{n-1}\right)$ est un polynome des coordonnées de $g_{1} \ldots g_{n-1}$ dont les coefficients, rationnels positifs, s'obtiennent de façon universelle à partir des coefficients de Taylor $\left(a_{v}^{i}\right)$, on vérifie que la $j^{\text {ème }}$ coordonnée $g_{n}^{j}(t)$ de $g_{n}(t)$ s'obtient comme combinaison linéaire d'intégrales du type:

$$
\begin{aligned}
\int_{s=0}^{l}\left(B_{s}^{J_{1}}\right)^{n_{1}} \ldots\left(B_{s}^{J_{l}}\right)^{n_{l}} d B_{s}^{i} & \text { avec } \sum_{i}^{l} n_{k}\left\|J_{k}\right\|=n-1 \text { si } i \neq 0 \\
& \text { et } \quad \sum_{i}^{l} n_{k}\left\|J_{k}\right\|=n-2 \text { si } i=0
\end{aligned}
$$

qui, par application itérée de la formule d'Ito, se ramène à des combinaisons linéaires à coefficients entiers positifs universels d'intégrales itérées

$$
\begin{array}{r}
\left(B_{t}^{J}\right) \text { telles que: }\|\boldsymbol{J}\|=\Sigma n_{k}\left\|J_{k}\right\|+1 \text { si } i \neq 0 \\
\text { et: }\|\boldsymbol{J}\|=\Sigma n_{k}\left\|J_{k}\right\|+2 \text { si } i=0,
\end{array}
$$

c'est-à-dire telles que: $\|J\|=n$.

Remarque. Si l'on introduit l'équation stochastique dépendant du paramètre $\varepsilon$ :

$$
\begin{aligned}
d X_{t}^{\varepsilon} & =\sum_{i=1}^{r} \varepsilon \sigma_{i}\left(X_{t}^{\varepsilon}\right) \circ d B_{t}^{i}+\varepsilon^{2} \sigma_{0}\left(X_{t}^{\varepsilon}\right) d B_{t}^{0} \\
X_{0}^{\varepsilon} & =x_{0} .
\end{aligned}
$$

Alors $\left(g_{k}(t)\right)$ est la série formelle de Taylor de $X_{t}^{\varepsilon}$ en $\varepsilon=0$, i.e.

$$
g_{k}(t)=\frac{1}{k !} \frac{d^{k}}{d \varepsilon^{k}} X_{\left.t\right|_{\varepsilon=0}}^{s} .
$$

Azencott a montré (théorème 1.6 [1]) que l'on a la formule de Taylor stochastique suivante:

Théorème 6. Si l'on définit le processus reste $R_{N+1}$ par

$$
X_{t}=x_{0}+\sum_{j=1}^{N} g_{j}(t)+t^{N+1 / 2} R_{N+1}(t)
$$


pour $t$ strictement inférieur au temps de vie $\zeta$ de $X_{t}$ et $R_{N+1}(t)=\partial$ point à l'infini pour $t \geqq \zeta$; alors $R_{N+1}$ est borné en probabilité lorsque $t$ tend vers 0 . C'est-à-dire $\lim _{t \rightarrow 0} P\left(\left|R_{N+1}\right| \geqq r\right)=0$ pour $r \geqq r_{0}$.

La preuve de ce résultat est fondée sur la propriété de scaling, de $X_{t}$ d'une part, et des $\left(g_{k}(t)\right)$ donnée par le $(2)$ de la proposition précédente. Ceci permet de ramener la formule de Taylor stochastique à une formule de Taylor usuelle en $\varepsilon$ de $X_{t}^{\varepsilon}$. Il reste ensuite à majorer le reste; nous renvoyons à Azencott pour cela.

Remarque. En fait, Azencott prouve le résultat avec une formulation Ito. Mais il est clair que les deux formulations sont équivalentes; en effet, toute équation d'Ito peut se transformer en une équation de Stratonovich et l'on a vu que les intégrales itérées d'Ito et de Stratonovich s'expriment comme combinaisons linéaires les unes des autres.

Rappelons ici que, grace à une majoration des moments du reste $R_{N+1}$, Azencott obtient les développements limités suivants:

Théorème 7. Soit $f$ une fonction $C^{\infty}$ bornée, on $a$ :

$$
E\left(f\left(X_{t}\right), t<\zeta\right)=f\left(x_{0}\right)+t L f\left(x_{0}\right)+\sum_{j=2}^{N} a_{j} t^{j}+O\left(t^{N+1}\right)
$$

où les $a_{j}$ sont des polynomes universels en les coefficients de Taylor en $x_{0}$ de $f$, des $\sigma_{i}$ et des scalaires $E\left(B_{1}^{J}\right)$.

Il suffit en effet de combiner la formule de Taylor usuelle pour $f$ et la formule de Taylor stochastique, puis d'utiliser la propriété de scaling des $\left(g_{k}(t)\right)$ ainsi qu'une propriété de symétrie évidente (pour vérifier que les coefficients des puissances demi-entières de $f$ s'annulent). Si l'on calcule les premiers termes, on vérifie que le coefficient de $\sqrt{t}$ vaut: $E\left(f^{\prime}\left(x_{0}\right) g_{1}(1)\right)$, c'est-à-dire $E\left(f^{\prime}\left(x_{0}\right) \sum_{i=1}^{r} \sigma_{i}\left(x_{0}\right) B_{1}^{i}\right)$ et donc qu'il est nul. Le terme suivant, coefficient de $t$ vaut:

$$
E\left(\frac{f^{\prime \prime}\left(x_{0}\right)}{2} \cdot g_{1}^{2}(1)+f^{\prime}\left(x_{0}\right) g_{2}(1)\right)=\frac{1}{2} L f\left(x_{0}\right)
$$

comme le montre un calcul très simple à partir des expressions de $g_{1}(t)$ et $g_{2}(t)$ :

$$
g_{1}(t)=\sum_{i=1}^{r} \sigma_{i}\left(x_{0}\right) B_{t}^{i} ; E\left(g_{2}^{i}(t)\right)=\sigma_{0}^{i}\left(x_{0}\right) t+\frac{1}{2} \sum_{k=1}^{r}\left[\sigma_{k}^{\prime}\left(x_{0}\right) \cdot \sigma_{k}\left(x_{0}\right)\right]^{i}
$$

Supposons, pour simplifier, que $X_{t}$ ait un temps de vie infini; on obtient ainsi un développement du semi-groupe $P_{t}$ de générateur $L$ agissant sur les fonctions $C^{\infty}$ :

$$
\frac{1}{t}\left(P_{t} f-f\right)=L f+\sum_{j=2}^{N} a_{j} t^{j}+O\left(t^{N+1}\right)
$$


(d) Convergence de la série de Taylor stochastique associée à une e.d.s. analytique

Supposons désormais que dans l'équation (1) les champs de vecteurs $\sigma_{i}$ sont analytiques au voisinage de $x_{0}$. Peut-on alors conclure à la convergence de la série de Taylor stochastique associée? Quel type de convergence?

Commençons par donner les deux exemples les plus simples d'une telle équation:

(1) Soit l'équation $\left\{\begin{array}{l}d X_{t}=X_{t} \circ d B_{t} \\ X_{0}=1\end{array}\right.$ alors, clairement, $X_{t}=e^{B_{t}}$, d'où $X_{t}=1$

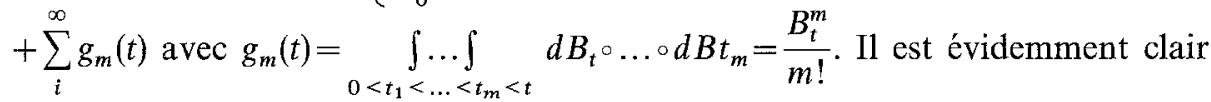
qu'ici la série de Taylor stochastique converge $\mathrm{p} \cdot \mathrm{s}$ pour tout $t$ et que $\Sigma\left|g_{m}(t)\right|_{L^{2}}$ converge pour tout $t$.

(2) Soit l'équation $\left\{\begin{array}{l}d X_{t}=X_{t}^{2} \circ d B_{t} \\ X_{0}=1\end{array}\right.$ alors $X_{t}=\frac{1}{1-B_{t}}$ d'où $X_{t}=1+\sum_{i}^{\infty} g_{m}(t)$ avec $g_{m}(t)=m ! \quad \int_{0<t_{1}<\ldots<t_{m}<t} d B_{t_{1}} \circ \ldots \circ d B t_{m}=B_{t}^{m}$. On a vu qu'alors la série $\sum_{1}^{\infty} g_{m}(t)$ ne converge $\mathrm{p} \cdot \mathrm{s}$ pour aucun $t$ et que $\Sigma\left|g_{m}(t)\right|_{L^{2}}$ diverge pour tout $t$, mais que $\sum_{1}^{\infty}\left|g_{m}(t)\right|$ converge $\mathrm{p} \cdot \mathrm{s}$ sur $\{\omega, t \leqq T(\omega)\}$ si $T$ est le temps d'arrêt $T=\inf \left(t,\left|B_{t}\right|=1\right)$.

Dans le cas général, on aura un résultat du type (2), à savoir convergence $\mathrm{p} \cdot \mathrm{s}$ avant un temps d'arrêt; les résultats du paragraphe 1 permettront de donner des critères qui assureront une convergence du type (1), à savoir une convergence $\mathrm{p} \cdot \mathrm{s}$ sur un intervalle déterministe et même une convergence $L^{2}$.

Commençons par montrer le résultat abstrait suivant: soit $C>0$, tel que les séries de Taylor des champs $\sigma_{i}$ convergent sur le pavé $] x_{0}-C, x_{0}+C\left[{ }^{n}\right.$. Pour $\rho>1$, introduisons le temps d'arrêt (éventuellement nul):

$$
\tau_{C}(\rho)=\inf \left(t, \sum_{m=1}^{\infty} \rho^{m}\left|g_{m}(t)\right| \geqq C\right)
$$

On a alors:

Théorème 8. Ps sur $t \leqq \tau_{\mathcal{C}}(\rho)$ on $a$ :

$$
X_{t}=x_{0}+\sum_{m=1}^{\infty} g_{m}(t)
$$

Preuve. Si $f(\varepsilon)$ est une fonction analytique sur le disque $\{\varepsilon \in \mathbb{C},|\varepsilon|<\rho\}$ alors pour $\rho^{\prime}<\rho$ on a:

$$
\forall p, \forall \varepsilon /|\varepsilon|<\rho^{\prime}\left|f(\varepsilon)-\sum_{0}^{p-1} \frac{f^{(k)}(0)}{k !} \varepsilon^{k}\right| \leqq \frac{M}{\rho^{\prime p}}
$$


$\operatorname{avec} M=\frac{1}{\left(1-\frac{\rho^{\prime}}{\rho}\right)} \sup _{|\varepsilon| \leq \rho^{\prime}}|f(\varepsilon)|$

Considérons ici la fonction $f(\varepsilon)=\sigma_{i}\left(x_{0}+\sum_{m=1}^{\infty} \varepsilon^{m} g_{m}(t)\right)$. Sur $t \leqq \tau_{c}(\rho) f(\varepsilon)$ est analytique sur $|\varepsilon|<\rho$ et

$$
\sup _{|\varepsilon| \leqq \rho^{\prime}}|f(\varepsilon)| \leqq \sup _{|x| \leqq C}\left|\sigma_{i}(x)\right|
$$

De plus, par définition des $C_{i}^{k}\left(g_{1} \ldots g_{k}\right)$, le développement de Taylor de $f$ en 0 à l'ordre $p$ est donné par $\sigma_{i}\left(x_{0}\right)+\sum_{k=1}^{p} C_{i}^{k}\left(g_{1} \ldots g_{k}\right) \varepsilon^{k}$ d'où

$$
\left|\sigma_{i}\left(x_{0}+\sum_{m=1}^{\infty} \varepsilon^{m} g_{m}(t)\right)-\left(\sigma_{i}\left(x_{0}\right)+\sum_{i}^{p} C_{i}^{k}\left(g_{1} \ldots g_{k}\right) \varepsilon^{k}\right)\right| \leqq \frac{K_{i}}{\rho^{\prime p}}
$$

pour tout $p$, tout $\varepsilon$ tel que $|\varepsilon|<\rho^{\prime}$ et $K_{i}=\frac{1}{1-\frac{\rho^{\prime}}{\rho}} \sup _{|x| \leqq C}\left|\sigma_{i}(x)\right|$. Or on a, par défini-
tion, des $g_{m}$ :

$$
\begin{aligned}
\sum_{1}^{p} \varepsilon^{m} g_{m}(t)= & \sum_{i=1}^{r} \sum_{n=1}^{p} \varepsilon^{m} \int_{0}^{t} C_{i}^{m-1}\left(g_{1}^{(s)} \ldots g_{m-1}^{(s)}\right) \circ d B_{s}^{i} \\
& +\sum_{m=2}^{p} \varepsilon^{m} \int_{0}^{t} C_{0}^{m-2}\left(g_{1}^{(s)} \ldots g_{m-2}^{(s)}\right) \circ d B_{s}^{0}
\end{aligned}
$$

D'où

$$
\sum_{i}^{p} \varepsilon^{m} g_{m}\left(t \wedge \tau_{c}(\rho)\right)=\sum_{i=1}^{r} \varepsilon \int_{0}^{t \wedge \tau_{c}} H_{i}^{p}(s) \circ d B_{s}^{i}+\varepsilon^{2} \int_{0}^{t \wedge \tau_{c}(\rho)} H^{p}(s) \circ d B_{s}^{i}
$$

avec

$$
\begin{aligned}
& H_{i}^{p}(s)=\sigma_{i}\left(x_{0}\right)+\sum_{k=1}^{p-1} \varepsilon^{k} C_{i}^{k}\left(g_{1}(s) \ldots g_{k}(s)\right) \\
& H_{0}^{p}(s)=\sigma_{0}\left(x_{0}\right)+\sum_{1}^{p-2} \varepsilon^{k} C_{i}^{k}\left(g_{1}(s) \ldots g_{k}(s)\right)
\end{aligned}
$$

Or, pour $|\varepsilon|<\rho^{\prime}$, on a:

$$
\begin{gathered}
\left\|\left[H_{i}^{p}(s)-\sigma_{i}\left(x_{0}+\sum_{m=1}^{\infty} \varepsilon^{m} g_{m}(t)\right)\right] 1_{t \leqq \tau_{c}(\rho)}\right\|_{L^{2}} \leqq \frac{k_{i}}{\rho^{\prime p}} P\left(t \leqq \tau_{c}(\rho)\right)^{1 / 2} \\
\left\|\left[H_{0}^{p}(s)-\sigma_{0}\left(x_{0}+\sum_{m=1}^{\infty} \varepsilon^{m} g_{m}(t)\right)\right] 1_{t \leqq \tau_{c}(\rho)}\right\|_{L^{2}} \leqq \frac{k_{0}}{\rho^{\prime p-1}} P\left(t \leqq t_{c}(\rho)\right)^{1 / 2}
\end{gathered}
$$


Si l'on a choisi $\rho^{\prime}>1$, les termes de droite de ces inégalités tendent vers 0 , lorsque $p \rightarrow \infty$, d'où on peut passer à la limite dans $(*)$; on obtient pour $|\varepsilon|<\rho^{\prime}:$

$$
\begin{aligned}
x_{0}+\sum_{1}^{\infty} \varepsilon^{m} g_{m}\left(t \wedge \tau_{c}(\rho)\right)= & x_{0}+\sum_{i=1}^{r} \varepsilon \int_{0}^{t \wedge \tau_{c}(\rho)} \sigma_{i}\left(x_{0}+\sum_{m=1}^{\infty} \varepsilon^{m} g_{m}(s)\right) \circ d B_{s}^{i} \\
& +\varepsilon^{2} \int_{0}^{t \wedge \tau_{c}(\rho)} \sigma_{0}\left(x_{0}+\sum_{1}^{\infty} \varepsilon^{m} g_{m}(s)\right) d B_{s}^{0}
\end{aligned}
$$

Ce qui montre que: $x_{0}+\sum_{1}^{\infty} g_{m}\left(t \wedge \tau_{c}(\rho)\right.$ est solution de la même équation que $X_{t \wedge \tau_{c}(\rho)}$, d'où le résultat.

Remarque. Ce résultat montre qu'avant le temps $\tau_{c}(\rho)$, la somme de la série de Taylor stochastique est bien $X_{t}$, mais n'assure pas que $\tau_{c}(\rho)$ est strictement positif et donc, pour l'instant, il pourrait être sans interet. Remarquons néanmoins que $\tau_{c}(\rho)$ est strictement positif $\mathrm{p} \cdot \mathrm{s}$ dès que $\tau(\rho)=\inf \left(t, \sum_{1}^{\infty} \rho^{k}\left|g_{k}(t)\right|\right.$ $=\infty)$ l'est.

Les résultats du $\S 1$ permettent de donner des critères sur les coefficients $P_{J}$ pour qu'il en soit ainsi:

Proposition 3. (1) $S i\left|P_{J}\right| \leqq K^{J}\|J\| !^{\alpha}$ pour tout $J$ tel que $|J| \geqq k_{0}$. Alors

$$
\begin{array}{ll}
\cdot \tau(r)=\infty & \text { si } \alpha<1 / 2 \\
\cdot \exists t_{1}>0 \quad \tau(r) \geqq t_{1} & \text { si } \alpha=1 / 2
\end{array}
$$

et donc $p \cdot s: \tau_{c}(\rho)>0$. Ainsi, le résultat du théorème est valable sur un intervalle stochastique $p \cdot s$ non vide.

(2) Si, de plus, les $\sigma_{i}$ sont entières, alors on a: $X_{t}=x_{0}+\sum_{1}^{\infty} g_{k}(t)$ pour tout $t$ si $\alpha<1 / 2$ et pour tout $t \leqq t_{1}$ si $a=1 / 2$.

Preuve. (1) Il s'agit simplement du corollaire 1.

(2) Les $\sigma_{i}$ étant entières, on a: $X_{t}=x_{0}+\sum_{i}^{\infty} g_{k}(t) p \cdot s$ sur $\left\{\omega, t \leqq \tau_{n}(\rho)\right\}$ pour tout $n \in \mathbb{N}$, donc $p \cdot s$ sur $\left\{\omega, \exists n t \leqq \tau_{n}(\rho)\right\}=\left\{\omega, \sum_{i}^{\infty} \rho^{k}\left|g_{k}(t)\right| \leqq n\right\}=\Omega$ si $\alpha<1 / 2$, ou si $\alpha=1 / 2$ et $t \leqq t_{1}$ d'où le résultat.

Ce résultat retrouve ainsi l'exemple 1) de l'équation

$$
\begin{aligned}
d X_{t} & =X_{t} \circ d B_{t} \\
X_{0} & =1 .
\end{aligned}
$$

Néanmoins, ce résultat fait intervenir les coefficients $P_{J}$ et non directement ceux des $\sigma_{i}$, et même avec des champs polynomiaux, il se peut que l'hypothèse ne soit pas vérifiée comme le montre l'exemple (2). 
Pour montrer qu'en général le résultat du théorème peut s'écrire sur un intervalle stochastique non vide, ou encore que le rayon de convergence aléatoire de la série de Taylor stochastique est $\mathrm{p} \cdot \mathrm{s}$ non nul, on va utiliser le résultat de dépendance analytique des paramètres.

Théorème 9. Il existe un temps d'arrét $T$ p.s strictement positif, tel que sur $[0, T[$ la série $\sum_{\mathbf{1}}^{\infty}\left|g_{k}(t)\right|$ converge $p \cdot s$, et

$$
X_{t}=x_{0}+\sum_{1}^{\infty} g_{k}(t)
$$

Preuve. Considérons l'équation

$$
\begin{aligned}
d X_{t}(\varepsilon) & =\sum_{i=1}^{r} \varepsilon \sigma_{i}\left(X_{t}(\varepsilon)\right) \circ d B_{t}^{i}+\varepsilon^{2} \sigma_{0}\left(X_{t}(\varepsilon)\right) d B_{t}^{0} \\
X_{0} & =x_{0} .
\end{aligned}
$$

Le théorème de dépendance analytique des paramètres démontré au $\S 2 \mathrm{~b}$ montre que, pour tout $\rho>0$, il existe un temps d'arrêt $\mathrm{p} \cdot \mathrm{s}$ strictement positif $T$, tel que sur $\left[0, T\left[X_{t}(\cdot)\right.\right.$ est analytique sur l'intervalle $]-\rho, \rho[$. Rappelons que l'on peut prendre: $T=\inf _{|\varepsilon|<\rho} T_{C}^{\varepsilon}, \varepsilon \in \mathbb{C}$ où $T_{C}^{\varepsilon}$ est le temps de sortie de la solution de l'équation $(*)$ complexifiée, hors du polydisque $\left\{z \in \mathbb{C}^{n},\left|z_{i}\right| \leqq C\right\}$ où les séries de Taylor des $\sigma_{i}$ en $x_{0}$ convergent. La série de Taylor en $\varepsilon=0$ de $X_{t}(\varepsilon)$ est donnée par les $g_{k}(t)$; on vérifie ainsi que si $t<T$ la série $\sum_{1}^{\infty} \varepsilon^{k} g_{k}(t)$ converge pour $|\varepsilon|<\rho$ et que $X_{t}(\varepsilon)=x_{0}+\sum_{1}^{\infty} \varepsilon^{k} g_{k}(t)$.

Il suffit de choisir $\rho>1$ pour conclure que: $\sum_{1}^{\infty}\left|g_{k}(t)\right|<\infty$ et que $X_{t}=X_{t}(1)$ $=x_{0}+\sum_{1}^{\infty} g_{k}(t)$.

Remarque. (1) Ce résultat affine le théorème 8 . En effet, on vérifie ici que $T \leqq \tau(\rho)$, donc que $\tau(\rho)>0 \mathrm{p} \cdot \mathrm{s}$ et donc que $\tau_{c}(\rho)>0 \mathrm{p} \cdot \mathrm{s}$. Mais ici le résultat est meilleur, puisque clairement $\tau_{c}(\rho) \leqq T$.

(2) Il était impossible sur l'équation stochastique (1) de complexifier le temps comme pour une équation ordinaire, l'introduction du paramètre $\varepsilon$ qui, lui, peut se complexifier a permis de tourner cette difficulté.

(3) La question de savoir précisément quel est le rayon de convergence de la série de Taylor stochastique, à savoir sur quel intervalle maximal $[0, T[$ la convergence a lieu est difficile. Ce temps maximal peut aussi bien ètre strictement plus petit que le temps de sortie de $X_{t}$ hors du domaine de convergence des $\sigma_{i}$ (comme le montre l'exemple 2) que strictement plus grand. 


\section{Flots stochastiques et ordinaires}

Il est bien connu (Bismut [4], Kunita [10]) qu'une équation stochastique à coefficients $C^{\infty}{ }_{b}$ définit un flot de difféomorphismes. Les développements de Taylor stochastiques précédents permettent de relier ces flots stochastiques et les flots d'équations différentielles ordinaires.

Soit $M$ une variété analytique; si $X$ est un champ de vecteur sur $M$, notons $\exp s X(\cdot)$ le flot associé à $X$. Soient $X_{0}, \ldots, X_{r} r+1$ champs de vecteurs analytiques définis sur un voisinage $U$ de $x_{0} \in M$. Pour $x \in U$, considérons le sous-espace vectoriel de $T_{x} M$ donné par $L(x)=\operatorname{Lie}\left(X_{0}, \ldots, X_{r}\right)(x)$. Soit alors $k=\operatorname{dim} L(x)$, et $\left(X^{K_{i}}\right)_{i=1 \ldots k}$ une base de $L\left(x_{0}\right)$, où l'on a noté $X^{K}$ le crochet des champs $X_{i}$ donné par $\left.X^{K}=X_{k_{1}},\left[X_{k_{2}} \ldots\left[X_{k_{m-1}}, X_{k_{m}}\right] \ldots\right]\right]$ si $K=\left(k_{1} \ldots k_{m}\right) \in\{0 \ldots r\}^{m}$.

Théorème 10. Soit $\xi_{t}\left(x_{0}\right)$ la solution de l'équation stochastique:

$$
\begin{aligned}
d \xi_{t} & =\sum_{i=0}^{r} X_{i}\left(\xi_{t}\right) \circ d B_{t}^{i} \\
\xi_{0} & =x_{0} .
\end{aligned}
$$

Alors, il existe un temps d'arrêt $T p \cdot s$ strictement positif et un champ de vecteur aléatoire $X_{t}(\omega)$ dans $L\left(x_{0}\right)$ tels que, sur $t<T$,

$$
\xi_{t}\left(x_{0}\right)=\exp X_{t}\left(x_{0}\right)
$$

Plus précisément, le champ $X_{t}$ s'écrit: $X_{t}=\sum_{i=1}^{k}\left(\sum_{m=1}^{\infty} \sum_{\|J\|=m} P_{J}^{i} B_{t}^{J}\right) X^{K} i$ où les $P_{J}^{i}$ sont des polynomes en les dérivées des champs $X_{i}$ en $x_{0}$.

Ainsi, ceci montre qu'en temps petit le flot stochastique est donné par le flot au temps 1 d'un champ de vecteur aléatoire dépendant du temps. Ce champ est une combinaison linéaire de crochets des $X_{i}$ dont les coefficients sont des sommes de séries de Taylor stochastiques. Résoudre l'équation stochastique revient donc à résoudre une équation différentielle ordinaire aléatoire dépendant du temps.

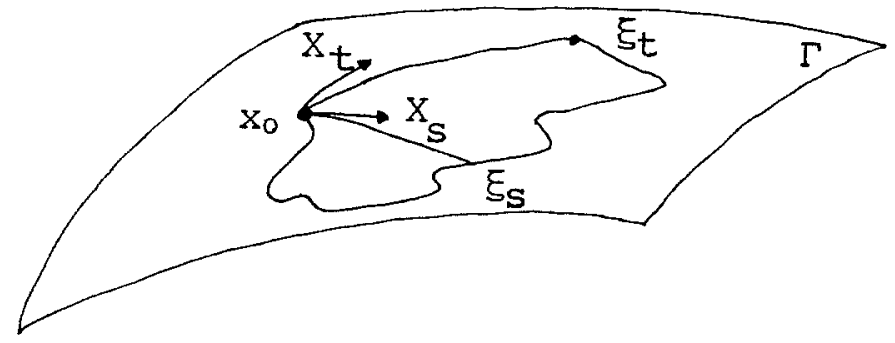


Preuve du théorème. Le théorème de Nagano [21] montre l'existence d'une variété analytique $\Gamma$, la feuille de Nagano de dimension $k$, telle que $x_{0} \in \Gamma$ et telle que $L(x)=T_{x} \Gamma$ pour $x \in \Gamma$ voisin de $x_{0}$.

Remarques. (1) Il n'est pas nécessaire ici de supposer que le rang de $L(x)$ est localement constant. Les différentes feuilles ne sont pas a priori de même rang. Le rang de $L(x)$ n'est constant que le long des feuilles.

(2) Il est clair, par le théorème du support de Stroock Varadhan que la diffusion $\xi_{t}$ issue de $x_{0}$ demeure en temps petit dans $\Gamma$. Soit l'application $\varphi$ $V \rightarrow \Gamma$

$$
\left(v_{1} \ldots v_{k}\right) \rightarrow \exp \left(\Sigma v_{i} X^{K_{i}}\right)\left(x_{0}\right)
$$

définie sur un voisinage $V$ de 0 dans $\mathbb{R}^{k}$ à valeurs dans $\Gamma$. Cette application est analytique sur $\boldsymbol{V}$. De plus, les dérivées de $\varphi$ en $v=0$ sont des polynomes universels en les dérivées des champs $X^{K_{i}}$ en $x_{0}$. En particulier:

$$
\frac{\partial}{\mid \partial V_{j}} \varphi l_{v=0}=X^{k_{j}}\left(x_{0}\right)
$$

Ainsi, quitte à restreindre $V$, ceci montre que $\varphi$ est un difféomorphisme de $V \operatorname{sur} \varphi(V)$.

Considérons alors sur $V$ les champs de vecteurs analytiques $Y_{i}=\varphi^{*} X_{i}$ et la solution $\zeta_{t}$ de l'équation

$$
\begin{aligned}
d \zeta_{t} & =\sum_{0}^{r} Y_{i}\left(\zeta_{t}\right) \circ d B_{t}^{i} \\
\zeta_{0} & =0 .
\end{aligned}
$$

La formule d'Ito montre que pour $t<$ temps de vie de $\zeta_{t}$ on a:

$$
\xi_{t}=\varphi\left(\zeta_{t}\right)=\exp \left(\sum_{1}^{k} \zeta_{t}^{i} \cdot X^{K_{i}}\right)\left(x_{0}\right)
$$

Il suffit alors d'appliquer le théorème 9 à la diffusion $\zeta_{t}$ pour conclure.

Remarques. (1) L'intervention de flots au temps 1 de champs dépendants du temps (ou transformées de Lie) est naturelle dans ce contexte (cf. AbrahamMarsden [20]).

(2) Le résultat obtenu n'est pas très précis quant à la description des $P_{J}$. En particulier, il est difficile de vérifier, par exemple, sous cette form qu'en fait, si l'algèbre de Lie $\left(X_{0} \ldots X_{r}\right)$ est $p$-nilpotente, les $P_{J}$ s'annulent pour $|J| \geqq p$, i.e., que les seules intégrales itérées d'ordre $\leqq p$ interviennent et que les sommes de séries de Taylor stochastiques sont des sommes finies.

(3) Moyennant une hypothèse supplémentaire naturelle, on peut donner une version $C^{\infty}$ du théorème: 
Théorème 11. Si $M$ est une variété $C^{\infty}$ et si les champs $X_{i}$ sont $C^{\infty}$ et tels que: au voisinage de $x_{0}$ le rang de $L(x)$ est constant. On a: $\exists T p \cdot s>0$ tq sur $t<T$.

$$
\xi_{t}=\exp X_{t}(\omega)\left(x_{0}\right)
$$

avec

$$
X_{t}(\omega)=\sum_{i=1}^{k}\left(\Sigma P_{J}^{i} B_{\varepsilon}^{J}\right) X^{k_{i}}+t^{N+1 / 2} R_{N}(t)
$$

et $R_{N}$ borné en probabilité lorsque t tend vers 0 .

La preuve est analogue en utilisant le théorème de Frobénius plutôt que celui de Nagano (ce qui est possible par l'hypothèse de rang constant) et le théorème 6 plutôt que le théorème 9 .

\section{Diffusions invariantes sur un groupe de lie}

\section{(a) Le cas general}

Dans le cas où la variété analytique $M$ est un groupe de Lie et où les $X_{i}$ sont des champs invariants, la description du champ aléatoire $X_{t}(\omega)$ du théorème 10 devient totalement explicite, et l'on va voir qu'un tel résultat recouvre les résultats de Yamato dans le cas nilpotent et se relie naturellement à ceux de Kunita dans le cas résoluble.

Soit $G$ un groupe de Lie connexe.

Soit $\mathscr{G}=T_{e} G$ son algèbre de Lie et exp l'application exponentielle sur $\mathscr{G}$. Notons $\mathscr{L}$ l'algèbre de Lie des champs de vecteurs sur $G$ invariants à gauche. (Tout ce que nous allons voir serait valable avec les champs invariants à droite). L'application $\mathscr{L} \rightarrow \mathscr{G}, X \rightarrow X(e)$ définit un isomorphisme d'algèbre de Lie. Soient $r+1$ champs de vecteurs $X_{0}, \ldots, X_{r} \in \mathscr{L}$.

Considérons alors l'équation stochastique invariante sur $G$ :

$$
\begin{aligned}
d \xi_{t} & =\sum_{i=0}^{r} X_{i}\left(\xi_{i}\right) \circ d B_{t}^{i} \\
\xi_{0} & =e .
\end{aligned}
$$

On sait que $\xi_{t}$ a un temps de vie infini; nous allons résoudre ici explicitement toute équation invariante (1) en temps petit.

Pour cela, introduisons quelques notations (cf. Bourbaki [5]).

Sur l'algèbre de Lie libre sur $\mathbb{Q}$ à $m$ lettres $L_{\mathbb{Q}}\left(U_{1}, U_{2}, \ldots, U_{m}\right)$. On introduit la série formelle de Campbell-Hausdorff par:

$$
H\left(U_{1}, \ldots, U_{m}\right)=\sum_{k \geqq 1} \frac{(-1)^{k-1}}{k} \sum_{p \in B_{k}} \frac{1}{|p| p !} U^{p}
$$


où l'on a noté

$$
B_{k}=\left\{\left(p_{i}^{j}\right)_{i \in\{1 \ldots m\}}, p_{i \in\{1 \ldots k\}}^{j} \in \mathbb{N}, \forall j \in\{1 \ldots k\} \sum_{i=1}^{m} p_{i}^{j}>0\right\}
$$

et pour $p \in B_{k}$ :

$$
\begin{aligned}
|p| & =\sum_{i, j} p_{i}^{j}, p !=\prod_{i, j} p_{i}^{j} ! \text { et } \\
U^{p} & =\left(\operatorname{ad} U_{1}\right)^{p 1} \ldots\left(\operatorname{ad} U_{m}\right)^{p_{m}^{1}}\left(\operatorname{ad} U_{1}\right)^{p_{1}^{2}} \ldots\left(\operatorname{ad} U_{m}\right)^{p_{m}^{2}} \\
& \ldots\left(\operatorname{ad} U_{1}\right)^{p \neq} \ldots\left(\operatorname{ad} U_{1}\right)^{p \uparrow} \ldots\left(\operatorname{ad} U_{m}\right)^{p_{m}^{k}-1} \cdot U_{m} .
\end{aligned}
$$

Considérons alors, si $J=\left(j_{1} \ldots j_{m}\right) \in\{0 \ldots r\}^{m}$, le terme $\beta_{J} m$-homogène de degré 1 en chaque variable dans la série $H\left(u_{j_{1}}, \ldots, u_{j_{m}}\right)$ où $u_{i}=X_{i}(e)$. On a clairement:

$$
\beta_{J}=\frac{1}{m} \sum_{k=1}^{m} \frac{(-1)^{k-1}}{k} \sum_{p \in \bar{B}_{k}} U_{J}^{p}
$$

où $\widehat{B}_{k}=\left\{p \in B_{k}, \forall i \in\{1 \ldots m\} \sum_{j=1}^{k} p_{i}^{j}=1\right\}$ et

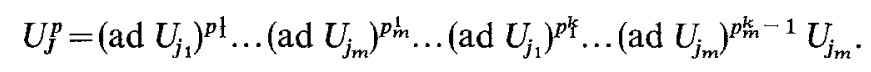

Par exemple, pour $|J|=1 J=(j)$, on a $\beta_{J}=u_{j}$.

Pour $|J|=2 J=(j, k)$, on a $\beta_{J}=\frac{1}{2}\left[u_{j}, u_{k}\right]$.

Pour $|J|=3 J=(i, j, k)$, on a $\beta_{J}=\frac{1}{3}\left[u_{k},\left[u_{j}, u_{i}\right]\right]-\frac{1}{6}\left[u_{j},\left[u_{k}, u_{i}\right]\right]$. On a alors le théorème principal de ce paragraphe.

Théorème 12. Il existe un temps d'arrêt $T$ p $\cdot s$ strictement positif, tel que, sur $\llbracket 0, T \llbracket$ la série $\sum_{m=1}^{\infty}\left|\sum_{\|J\|=m} \beta_{J} B_{t}^{J}\right|$ converge presque surement, et:

$$
\xi_{t}=\exp \left(\sum_{m=1}^{\infty} \sum_{\|J\|=m} \beta_{J} B_{t}^{J}\right)
$$

Ce théorème donne ainsi une résolution explicite en carte exponentielle de toute équation stochastique invariante sur un groupe de Lie (en temps petit). $\xi_{t}$ est l'exponentielle d'une fonction universelle de la «diffusion universelle» $\left(B_{t}^{J}\right)$ et des crochets des $X_{i}$. Remarquons le fait important que les coefficients des intégrales d'ordre $k$ sont des crochets du même ordre.

Preuve. Soit $U$ l'ouvert de $\mathscr{G}$ constitué des points où l'exponentielle est régulière. Si $s(x)$ désigne le spectre de ad $x$ (considéré comme endomorphisme de $\mathscr{G}$ complexifiée), on sait que $U=\{x \in \mathscr{G}, s(x) \cap 2 \pi \mathbb{Z} \subset\{0\}\}$.

Soit $\sigma_{i}$ le champ de vecteur sur $U$ donné par

$$
\sigma_{i}(x)=\frac{\operatorname{ad}(-x)}{e^{\text {ad }-x}-I}
$$


où $\frac{\operatorname{ad}(-x)}{e^{\mathrm{ad}(-x)}-I}$ est un abus de notation pour $\left(\sum_{p=0}^{\infty} \frac{1}{(p+1) !} \mathrm{ad}(-x)^{p}\right)^{-1}$ (ce qui a bien un sens pour $x \in U$ ).

On a alors, pour $x \in U$ :

$$
\left(T_{x} \exp \right)\left[\sigma_{i}(x)\right]=X_{i}(\exp x) .
$$

En effet, pour $h \in T_{x} U$ identifié à $\mathscr{G}$, on a

$$
T_{x} \exp (h)=\exp x \cdot \sum_{0}^{\infty} \frac{1}{(p+1) !}(\operatorname{ad}-x)^{p} \cdot h .
$$

D'où, pour $h=\sigma_{i}(x)$

$$
\begin{aligned}
T_{x} \exp \left[\sigma_{i}(x)\right] & =\exp x \cdot u_{i}=\exp x \cdot X_{i}(e) \\
& =X_{i}(\exp x) \quad \text { par l'invariance de } X_{i} .
\end{aligned}
$$

Soit alors l'équation sur $U$ associée aux $\sigma_{i}$ :

$$
\begin{aligned}
d \zeta_{t} & =\sum_{i=0}^{r} \sigma_{i}\left(\zeta_{t}\right) \circ d B_{t}^{i} \\
\zeta_{0} & =0 .
\end{aligned}
$$

Si $T_{1}$ désigne le temps de vie de $\zeta_{t}$, la formule d'Ito et la relation (*) montrent que, sur $\llbracket 0, T_{1} \llbracket, \xi_{t}=\exp \zeta_{t}$.

D'autre part, les champs $\sigma_{i}$ sont analytiques sur $U$. Plus précisément, la série de Taylor de $\sigma_{i}$ en 0 est donnée par:

$$
\left(\mathrm{Id}-\frac{\mathrm{ad}-x}{2}+\sum_{n=1}^{\infty} \frac{(-1)^{n-1}}{2 n !} b_{n}(\mathrm{ad}-x)^{2 n}\right) \cdot U_{i}
$$

où $b_{n}$ désigne le $n^{\text {ème }}$ nombre de Bernouilli. Cette série est sommable sur l'ouvert $V \subset U: V=\{x \in \mathscr{G}$, le rayon spectral de ad $x<2 \pi\}$. En effet, si $W$ est l'ouvert de $L(\mathscr{G})$ (l'ensemble des endomorphismes de $\mathscr{G})$ donné par $W=\{A \in L(\mathscr{G})$, spectre $(A) \cap 2 \pi \mathbb{Z} \subset\{0\}\}$ et, si $\phi$ désigne l'application de $W$ dans $L(\mathscr{G})$ donnée par $\phi(A)=\frac{A}{e^{A}-I}, \phi$ est analytique sur $W$ et sa série de Taylor en 0 est donnée par:

$$
\phi(A)=\mathrm{Id}-\frac{A}{2}+\sum_{i}^{\infty} \frac{(-1)^{n-1}}{2 n !} b_{n} A^{2 n}
$$

Cette série est normalement convergente si le rayon spectral de $A$ est strictement inférieur à $2 \pi$, car, dans ce cas, pour $n$ assez grand, on a: $\left\|A^{n}\right\| \leqq C^{n}$ avec $C<2 \pi$ et la série réelle $1-\frac{x}{2}+\Sigma \frac{(-1)^{n-1}}{2 n !} b_{n} x^{2 n}$ est convergente pour $|x|<2 \pi$ (et a 
pour some $\frac{x}{e^{x}-1}$ : ceci pouvant servir de définition aux nombres de Bernouilli $\left.b_{n}\right)$.

Il suffit alors d'appliquer le théorème $9 \mathrm{du} \S 1$ pour obtenir le développement de $\zeta_{t}$ en série de Taylor stochastique. Il reste seulement à identifier les termes du développement:

Considérons le système $\left(\mathscr{S}_{m}\right)$ associé à l'équation (2):

$$
\begin{aligned}
d g_{1}(t) & =\sum_{i=1}^{r} u_{i} d B_{t}^{i} \\
& \vdots \\
d g_{m}(t) & =\sum_{i=1}^{r} C_{i}^{m-1}\left(g_{1}(t) \ldots g_{m-1}(t)\right) \circ d B_{t}^{i}+C_{0}^{m-2}\left(g_{1}(t) \ldots g_{m-2}(t) \circ d B_{t}\right.
\end{aligned}
$$

où $C_{i}^{k}\left(g_{1} \ldots g_{k}\right)$ est le coefficient de $x^{k}$ dans la série de Taylor en 0 de $\sigma_{i}\left(\sum_{i}^{k} x^{l} g_{l}\right)$. On a alors la description cherchée des $g_{k}$ qui achève la preuve du théorème: (La preuve de ce lemme est renvoyée en appendice.)

Lemme 4. Pour tout $k$ :

(a) $g_{k}(t)=\sum_{\|J\|=k} \beta_{J} B_{t}^{J}$,et

(b) $C_{i}^{k}\left(g_{1}(t) \ldots g_{k}(t)\right)=\sum_{\|J\|=k} \beta_{J \cup\{i\}} B_{i}^{J}$.

en notant $J \cup\{i\}=\left(j_{1} \ldots j_{m}, i\right)$ si $J=\left(j_{1} \ldots j_{m}\right)$.

Remarque. Le résultat que nous venons de prouver est local et, comme tel, n'utilise que la structure de groupe de Lie local associé à l'algèbre $\mathscr{G}$. Cette remarque sera utilisée plus loin.

\section{(b) Le cas nilpotent}

Dans le cas où l'algèbre engendrée par les champs $X_{i}$ est nilpotente, le théorème général se simplifie.

Théorème 13. Si Lie $\left(X_{0}, \ldots, X_{r}\right)$ est p-nilpotente, on a, presque surement, pour tout $t \geqq 0$

$$
\xi_{t}=\exp \left(\sum_{m=1}^{p} \sum_{|J|=m} \beta_{J} B_{t}^{J}\right)
$$

Preuve. Dans le cas nilpotent, l'exponentielle est régulière sur $\mathscr{G}$. Les champs

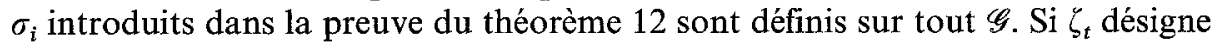
la solution sur $\mathscr{G}$ de l'équation:

$$
\begin{aligned}
d \zeta_{t} & =\sum_{i=0}^{r} \sigma_{i}\left(\zeta_{t}\right) \circ d B_{t}^{i} \\
\zeta_{0} & =0
\end{aligned}
$$


la série de Taylor stochastique de $\zeta_{t}$ est en fait une somme finie $\sum_{m=1}^{p} \sum_{|J|=m} \beta_{J} B_{t}^{J}$ et, par le théorème $8, \mathrm{du} \S 2$ (ici évident), on vérifie que

$$
\forall t \geqq 0 \quad \zeta_{t}=\sum_{m=1}^{p} \sum_{|J|=m} \beta_{J} B_{t}^{J}
$$

D'autre part, la formule d'Ito, appliquée à l'exponentielle, montre comme précédemment que $\exp \zeta_{t}$ est solution de l'équation (1), pour tout $t$, d'où, par unicité, on obtient le théorème.

\section{Exemples}

(1) Le cas abélien

Si Lie $\left(X_{0}, \ldots, X_{r}\right)$ est abélienne, le résultat se simplifie encore pour donner:

$$
\xi_{t}=\exp \left(\sum_{i=0}^{r} u_{i} B_{t}^{i}\right) \quad \text { avec } u_{i}=X_{i}(e)
$$

Dans ce cas, $\xi_{t}$ est une fonction régulière du mouvement brownien $\left(B_{t}^{1}, \ldots, B_{t}^{r}\right)$ et du temps. On retrouve ainsi les résultats de Doss [6] et Sussmann [17].

Il faut remarquer que la formule ainsi obtenue est triviale, et que le travail précédent n'est pas nécessaire ici. Il suffit en effet d'utiliser la formule d'Ito pour constater que $\exp \left(\sum_{0}^{r} u_{i} B_{\mathrm{r}}^{i}\right)$ est solution de l'équation (1).

\section{(2) Le groupe d'Heisenberg}

Soit $\mathbb{H}_{1}$ le groupe des matrices triangulaires supérieures, à coefficients réels, avec des 1 sur la diagonale, de taille $3 \times 3$ :

$$
\begin{array}{cc}
H_{1}=\left\{\left(\begin{array}{ccc}
1 & x & z \\
0 & 1 & y \\
0 & 0 & 1
\end{array}\right)(x, y, z) \in \mathbb{R}^{3}\right\} . \\
\text { Soit } u_{1}=\left(\begin{array}{lll}
0 & 1 & 0 \\
0 & 0 & 0 \\
0 & 0 & 0
\end{array}\right) & u_{2}=\left(\begin{array}{lll}
0 & 0 & 0 \\
0 & 0 & 1 \\
0 & 0 & 0
\end{array}\right) \quad u_{3}=\left(\begin{array}{lll}
0 & 0 & 1 \\
0 & 0 & 0 \\
0 & 0 & 0
\end{array}\right)
\end{array}
$$

$u_{1}, u_{2}, u_{3}$ constituent une base de l'algèbre de Lie de $\mathbb{H}_{1}$ et l'on a $\left[u_{1}, u_{2}\right]=u_{3}$ les autres crochets étant nuls. 
Si $\xi_{t}$ désigne la solution sur $\mathbb{H}_{1}$ de l'équation

$$
\begin{aligned}
d \xi_{t} & =\sum_{i=1}^{2} X_{i}\left(\xi_{t}\right) \circ d B_{t}^{i} \\
\xi_{0} & =l
\end{aligned}
$$

où $X_{i}$ désigne le champ invariant à gauche sur $\mathbb{H}_{1}$ tel que $X_{i}(l)=u_{i}$. On a

$$
\begin{gathered}
\xi_{t}=\left(\begin{array}{ccc}
1 & B_{t}^{1} & \int_{0}^{t} B_{s}^{1} d B_{s}^{2} \\
0 & 1 & B_{t}^{2} \\
0 & 0 & 1
\end{array}\right) \text { d'où: } \\
\xi_{t}=\exp \left(B_{t}^{1} u_{1}+B_{t}^{2} u_{2}+\left(\int_{0}^{t} B_{s}^{1} d B_{s}^{2}-\frac{1}{2} B_{t}^{1} B_{t}^{2}\right) u_{3}\right) \\
\xi_{t}=\exp \left(B_{t}^{1} u_{1}+B_{t}^{2} u_{2}+\frac{1}{2}\left(\int_{0}^{t} B_{s}^{1} d B_{s}^{2}-\int_{0}^{t} B_{s}^{2} d B_{s}^{1}\right)\left[u_{1}, u_{2}\right]\right)
\end{gathered}
$$

On a ainsi retrouvé directement, dans ce cas, le résultat annoncé. Le coefficient de $\int_{0}^{t} B_{s}^{1} d B_{s}^{2}\left(\right.$ resp. $\left.\int_{0}^{t} B_{s}^{2} d B_{s}^{1}\right)$ est bien comme prévu $\frac{1}{2}\left[u_{1}, u_{2}\right]$ (resp. $\left.-\frac{1}{2}\left[u_{1}, u_{2}\right]=\frac{1}{2}\left[u_{2}, u_{1}\right]\right)$ à savoir $\beta_{(1,2)}\left(\operatorname{resp} . \beta_{(2,1)}\right)$.

Ce résultat est bien sûr bien connu (cf. Gaveau [8]).

Dans le cas nilpotent, il est possible de donner une autre preuve du théorème en suivant l'idée de Kunita [11], qui consiste à appliquer directement la formule de Campbell-Hausdorff à la suite des approximations de $\xi_{t}$ dite des intégrales stochastiques multiplicatives (cf. Ibéro [9]).

Nous allons donner ici cette preuve pour montrer comment aboutir à un développement de $\xi_{t}$ exactement identique à celui obtenu.

Pour cela, précisons certains faits sur la série de Campbell-Hausdorff. On a déjà introduit la série de Campbell-Hausdorff à $n$ variables: $H\left(U_{1} \ldots U_{n}\right)$ dans l'algèbre de Lie libre $L_{\mathbb{Q}}\left(u_{1}, \ldots, u_{n}\right)$. Le commutateur $U^{I}=\left[U_{i_{1}}\left[U_{i_{2}} \ldots U_{i_{n-1}}, U_{i_{n}}\right]\right]$ apparaît plusieurs fois dans la série $H\left(U_{1}, \ldots, U_{n}\right)$. Regroupons les termes où il apparaît et appelons $C_{I}$ le coefficient affectant $U^{I}$, de telle sorte que:

$$
H\left(U_{1}, \ldots, U_{n}\right)=\sum_{m=1} \sum_{I \in\{1 \ldots n\}^{m}} C_{I} U^{I}
$$

Nous allons montrer que, pour de nombreux $I$, les $C_{I}$ coïncident. Soient $\left(x_{1}, \ldots, x_{m}\right) \in\left(\mathbb{R}^{+*}\right)^{m}$ et $\left(y_{1}, \ldots, y_{m}\right) \in\left(\mathbb{R}^{+*}\right)^{m}$; on dira que $\left(x_{1}, \ldots, x_{m}\right) \sim\left(y_{1}, \ldots, y_{m}\right)$ s'il existe une bijection strictement croissante $\phi$ de $\left\{x_{1}, \ldots, x_{m}\right\}$ dans $\left\{y_{1}, \ldots, y_{m}\right\}$ telle que $\phi\left(x_{i}\right)=y_{i} \forall i$. Il est clair que $\sim$ est une relation d'équivalence sur $\left(\mathbb{R}^{+*}\right)^{m}$. On peut décrire précisément l'ensemble quotient $A_{m}=\left(\mathbb{R}^{+*}\right)^{m} / \sim:$ Si $n_{1}, \ldots, n_{k}$ 
sont $k$ entiers, tels que $\sum_{1}^{k} n_{i}=m$, notons $\sigma_{n_{1} \ldots n_{k}}$ le sous-groupe du groupe symétrique $\sigma_{m}$ isomorphe à $\sigma_{n_{1}} \times \ldots \times \sigma_{n_{\mathrm{k}}}$ donné par

$$
\sigma_{n_{1} \ldots n_{k}}=\left\{\tau \in \sigma_{m} / \forall i \quad \tau\left(\left\{n_{i}+1, \ldots, n_{i+1}\right\}\right)=\left\{n_{i}+1, \ldots, n_{i+1}\right\}\right\} .
$$

Si $\sigma \in \sigma_{m}$, on notera $\sigma$ sa classe modulo $\sigma_{n_{1} \ldots n_{k}}$.

Et on posera:

$$
\begin{aligned}
\lambda\left(n_{1} \ldots n_{k}, \delta\right) & =\left\{\left(t_{1} \ldots t_{m}\right) \in \mathbb{R}^{+* m} / t_{\sigma(1)}=\ldots=t_{\sigma(n)}<t_{\sigma(n+1)}\right. \\
& \left.=\ldots=t_{\sigma\left(n_{2}\right)}<\ldots<t_{\sigma\left(\sum_{1}^{n-1} n_{i}+1\right)}=\ldots=t_{\sigma(m)}\right\} .
\end{aligned}
$$

Il est clair que cette définition ne dépend pas du choix du représentant de $\stackrel{\circ}{\sigma}$. Alors tout $\lambda$ de $A_{m}$ s'écrit de façon unique comme un $\lambda\left(n_{1} \ldots n_{k}, \sigma\right)$. On appellera degré de $\lambda\left(n_{1} \ldots n_{k}, \stackrel{\circ}{\sigma}\right)$ l'entier $\max _{1 \leqq i \leqq k}\left(n_{i}\right)$. Les éléments $\lambda$ de $A_{m}$ de degré 1 sont les cones ouverts de $\left(\mathbb{R}^{+*}\right)^{m}$ :

$$
\lambda(\sigma)=\left\{\left(t_{1} \ldots t_{m}\right) \in \mathbb{R}^{+* m} / t_{\sigma(1)}<\ldots<t_{\sigma(m)}\right\} .
$$

Les éléments de degrés supérieurs sont des cones obtenus par intersection de la frontière de ces $\lambda(\sigma)$ avec des sous-espaces de $\mathbb{R}^{m}$ de dimension strictement inférieure à $m$.

On a alors: Proposition 4. (1) Si $\lambda \in A_{m}$ et si $\left\{\begin{array}{l}I \in\{1 \ldots n\}^{m} \cap \lambda \\ I^{\prime} \in\left\{1 \ldots n^{\prime}\right\}^{m} \cap \lambda\end{array}\right.$ alors $C_{I}=C_{I^{\prime}}$ que l'on notera
$C_{\lambda}$.

(2) Si $\lambda\left(n_{1} \ldots n_{k}\right)$, o̊) est de degré 2 , on a

$$
C_{\lambda\left(n_{1} \ldots n_{k}, \breve{\sigma}\right)}=\frac{1}{2^{m-k}} \sum_{\sigma \in \dot{\sigma}} C_{\lambda(\sigma)}
$$

Preuve. Le (1) est évident.

(2) La preuve se fait par récurrence sur le nombre $l=m-k$ de $n_{i}$ égaux à 2. L'identité est évidente avec $l=0$. Pour $l>0$, ou $k<m$, supposons (sans restreindre la généralité) que la classe d'équivalence $\sigma$ soit celle du neutre, soit $I=\left(i_{1}, \ldots, i_{m}\right) \in \lambda\left(n_{1} \ldots n_{k}, \stackrel{\sigma}{\sigma}\right)$ on a donc:

$i_{1}=i_{2}=\ldots=i_{n_{1}}=l_{1}<i_{n_{1}+1}=\ldots=i_{n_{1}+n_{2}+1}=l_{2}<\ldots<i_{n_{1}+\ldots+{ }_{k-1}+1}=\ldots=i_{m}=l_{k}$.

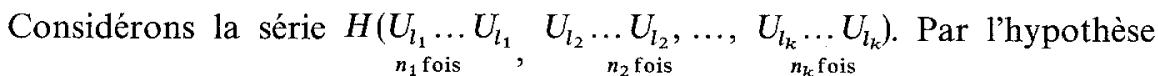
de récurrence, on sait que, dans cette série, le crochet $U^{n_{2}}$ apparaît muni du coefficient :

$$
n_{1} ! \ldots n_{k} !\left(C_{\lambda\left(n_{1} \ldots n_{k}, \sigma\right)}+\left(\frac{1}{2}+\frac{1}{4}+\ldots+\frac{1}{2^{m-k}}\right) \sum_{\sigma \in \check{\sigma}} C_{\lambda(\sigma)}\right)
$$


qui vaut:

$$
2^{m-k} C_{\lambda\left(n_{1} \ldots n_{k}, \breve{\sigma}\right)}+\left(2^{m-k}-1\right) \sum_{\sigma \in \check{\sigma}} C_{\lambda(\sigma)},
$$

car $n_{1} ! \ldots n_{k} !=2^{m-k}$, puisque $n_{i} \in\{1,2\}$.

Or la série $H\left(U_{l_{1}} \ldots U_{l_{1}}, \ldots, U_{l_{k}} \ldots U_{l_{k}}\right)$ est égale à la série $H\left(n_{1} U_{l_{1}}, \ldots, n_{k} U_{l_{k}}\right)$ où le crochet $U^{I}$ apparaît avec le coefficient:

$$
n_{1}^{n_{1}} \ldots n_{k}^{n_{k}} C_{\lambda\left(n_{1} \ldots n_{k}, \check{\sigma}\right)}=2^{2(m-k)} C_{\lambda\left(n_{1} \ldots n_{k}, \check{\sigma}\right)} .
$$

D'où l'on tire

$$
2^{2(m-k)} C_{\lambda\left(n_{1} \ldots n_{k}, \check{\sigma}\right)}=2^{m-k} C_{\lambda\left(n_{1} \ldots n_{k}, \check{\sigma}\right)}+\left(2^{m-k}-1\right) \sum_{\sigma \in \check{\sigma}} C_{\lambda(\sigma)} .
$$

d'où le résultat.

On peut alors prouver le

Théorème 14. Si Lie $\left(X_{0} \ldots X_{r}\right)$ est p-nilpotente, on a, presque surement, pour tout $t$

où l'on a posé

$$
\xi_{t}=\exp \sum_{m=1}^{p-1} \sum_{|J|=m} \alpha_{t}^{J} U^{J}
$$

$$
\alpha_{t}^{J}=\sum_{\substack{\lambda \in \Lambda_{m} \\ d^{0} \lambda \leqq 2}} C_{\lambda\left(n_{1} \ldots n_{k}, \check{\sigma}\right)} I_{t}\left(B^{j \sigma(1)} \ldots B^{j \sigma(m)},\left(n_{1} \ldots n_{k}\right)\right)
$$

Rappelons que la définition de l'intégrale itérée d'Ito $I_{t}$, obtenue en remplaçant, dans l'intégrale itérée $\int_{0<t_{1}<\ldots<t_{m}<t} d B_{t}^{j \sigma(1)} \ldots d B_{t_{m}}^{j \sigma(m)}$, certains des $d B^{j} d B^{k}$ par des $d\left\langle B^{j}, B^{k}\right\rangle$, est donnée au $\S 1$.

Preuve. Considérons la suite $\xi_{t}^{(n)}$ qui définit $\xi_{t}$ comme une intégrale stochastique multiplicative (Ibéro [9]),

$$
\xi_{t}^{(n)}=\exp V_{2^{n}}^{(n)} \ldots \exp V_{1}^{(n)}
$$

où $V_{k}^{(n)}=\sum_{j=0}^{r} \delta^{(n)} B_{k}^{j} U_{j}$ avec $\delta^{(n)} B_{k}^{j}=B_{t_{k+1}}^{j}-B_{t_{k}}^{j}$ et $t_{k}=\frac{k}{2^{n}} t$. On sait que $\xi_{t}^{(n)}$ converge en probabilité vers $\xi_{t}$.

Or sur l'algèbre nilpotente $\mathscr{G}$, on a la formule de Campbell-Hausdorff $\forall V_{1} \ldots V_{m} \in \mathscr{G} \exp V_{m} \ldots \exp V_{1}=\exp H\left(V_{1} \ldots V_{m}\right)$. Or

$$
\begin{aligned}
H\left(V_{1}^{(n)}, \ldots, V_{2^{n}}^{(n)}\right. & =\sum_{m=1}^{p-1} \sum_{\lambda \in A_{m}} C_{\lambda}\left(\sum_{I \in\{1 \ldots 2 n\}^{m} \cap \lambda} V^{I}\right) \\
& =\sum_{m=1}^{p-1} \sum_{\lambda \in \Lambda_{m}} C_{\lambda} \sum_{J \in\{0 \ldots r\}^{m}} \sum_{I \in\left\{1 \ldots 2^{n ! m} \cap \lambda\right.}\left(\delta^{(n)} B_{i_{1}}^{j_{1}} \ldots \delta^{(n)} B_{i_{m}}^{j m}\right) U^{J} .
\end{aligned}
$$

Or le lemme suivant est élémentaire:

Lemme 5. (1) Si $\lambda=\lambda\left(n_{1} \ldots n_{k}, \stackrel{\circ}{)}\right)$ est de degré inférieur ou égal à 2, la quantité $\sum_{I \in\left\{1 \ldots 2^{n\}^{m} \cap \lambda}\right.} \delta^{(n)} B_{i}^{j} \ldots \delta^{(n)} B_{i_{m}}^{j_{m}}$ converge $L^{2}$ vers l'intégrale itérée d'Ito: $I_{t}\left(B^{j \sigma(1)} \ldots B^{j \sigma(m)}, n_{1} \ldots n_{k}\right)$. 
(2) Si $\lambda$ est de degré supérieur ou égal à 3, la même quantité converge vers 0 .

D'où l'on tire que $H\left(V_{1}^{(n)} \ldots V_{2^{n}}^{(n)}\right)$ converge en probabilité vers:

$$
\sum_{m=1}^{p-1} \sum_{J \in\{0 \ldots r\}^{m}} U^{J} \sum_{\substack{\lambda \in A m \\ d^{\circ} \lambda \leqq 2}} C_{\lambda} I_{t}\left(B^{j \sigma(1)} \ldots B^{j \sigma(m)}, n_{1} \ldots n_{k}\right) .
$$

c'est-à-dire vers:

$$
\sum_{m=1}^{p-2} \sum_{J \in\{0 \ldots r\}^{m}} \alpha_{t}^{J} U^{J} . \quad \text { C.Q.F.D. }
$$

Remarque. Meyer a introduit ([14] p. 324-325) une notion très générale d'intégrales stochastiques multiples; il constatait alors que cette notion n'avait jamais servi. En fait ici, ces intégrales apparaissent naturellement.

Théorème 15. Le coefficient $\alpha_{t}^{J} d u$ commutateur $U^{J}$ dans le développement précédent est l'intégrale multiple au sens de Meyer [14] de la fonction déterministe $\sum_{\lambda \in A_{m}} C_{\lambda} 1_{\lambda}\left(t_{1} \ldots t_{m}\right)$ sur $[0, t]^{m}$ par rapport à la mesure produit $d B_{t_{1}}^{j_{1}} \ldots d B_{t_{m}}^{j_{m}}$.

Preuve. On renvoie ici aux pp. 324-325 du cours de Meyer, pour la définition de ces intégrales multiples. La partition de $[0, t]^{m}$ induite par notre relation d'équivalence $\sim$ correspond exactement aux dégénérescences que considère Meyer et, pour chaque $\lambda$ de degré inférieur ou égal à 2 , l'intégrale $I_{t}\left(B^{j \sigma(1)} \ldots B^{j \sigma(m)}, n_{1} \ldots n_{k}\right)$ correspond à l'intégrale introduite par Meyer. Les $\lambda$ de degré supérieur ou égal à 3 n'interviennent pas, du fait de la continuité $\operatorname{des} B_{t}^{j}$. D'où le résultat.

Le développement obtenu au théorème ne constitue pas encore un développement en série de Taylor stochastique; pour cela, il faut exprimer les intégrales multiples $\alpha_{t}^{J}$ à partir d'intégrales itérées de Stratonovich. Précisément, on a:

\section{Théorème 16.}

$$
\alpha_{t}^{J}=\sum_{\sigma \in \sigma_{m}} C_{\lambda(\sigma)} \int_{0<t_{1}<\ldots<t_{m}<t} \ldots \int_{t_{1}}^{j \sigma(1)} \circ \ldots \circ d B_{t m}^{j \sigma(m)}
$$

Dans la preuve de ce théorème qui permettra d'identifier les développements de $\xi_{t}$ obtenus par nos deux preuves (théorème 13 et théorème 14 ), il se produit un «miracle», à savoir la combinaison parfaite de la proposition 4 entièrement algébrique et la relation de la proposition 1 entre intégrales itérées d'Ito et de Stratonovich purement probabiliste.

Preuve. $\lambda\left(n_{1} \ldots n_{k}, \stackrel{\circ}{\sigma}\right)$ est de degré au plus 2 si $n_{1}+\ldots+n_{k}=m$ et $n_{i} \in\{1,2\}$ ou encore, avec les notations du paragraphes 1 , si $n_{1} \ldots n_{k} \in A_{m}^{k}$ d'où,

$$
\begin{aligned}
\alpha_{t}^{J}= & \sum_{\substack{\lambda \in \lambda m \\
d^{0} \lambda \leqq 2}} C_{\lambda} I_{t}\left(B^{j \sigma(1)} \ldots B^{j \sigma(m)}, n_{1} \ldots n_{k}\right) \\
& \sum_{k=[m / 2]}^{m} \sum_{n_{1} \ldots n_{k} \in A_{m}^{k}} \sum_{\sigma \in \sigma \in m / \sigma n_{1} \ldots n_{k}} C_{\lambda\left(n_{1} \ldots n_{k}, \check{\sigma}\right)} I_{t}\left(B^{j \sigma(1)} \ldots B^{j \sigma(m)}, n_{1} \ldots n_{k}\right) .
\end{aligned}
$$


D'où, par le lemme, on a:

$$
\begin{aligned}
\alpha_{t}^{J} & =\sum_{k=\left[\begin{array}{l}
m \\
2
\end{array}\right]} \frac{1}{2^{m-k}} \sum_{n_{1} \ldots n_{k} \in A_{m}^{k}} \sum_{\delta} \sum_{\sigma \sigma \sigma / \sigma n_{1} \ldots n_{k}} \sum_{\sigma \in \delta} C_{\lambda(\sigma)} I_{t}\left(B^{j \sigma(1) \ldots b j \sigma(m)}, n_{1} \ldots n_{k}\right) \\
& =\sum_{\sigma \in \sigma m} C_{\lambda(\sigma)} \sum_{k=\left[\begin{array}{c}
m \\
2
\end{array}\right]}^{m} \frac{1}{2^{m-k}} \sum_{n_{1} \ldots n_{k} \in A_{m}^{k}} I_{t}\left(B^{j \sigma(1)} \ldots B^{j \sigma(m)}, n_{1} \ldots n_{k}\right),
\end{aligned}
$$

ce qui est précisément égal, par la proposition 1 , à

$$
\alpha_{t}^{J}=\sum_{\sigma \in \sigma_{m}} C_{\lambda(\sigma)} \int_{0<t_{1}<\ldots<t_{m}<t} d B_{t_{1}}^{j \sigma(1)} \ldots \ldots \circ d B_{t_{m}}^{j \sigma(m)}
$$

Ceci permet donc d'écrire le développement obtenu au théorème 14, sous la forme:

$$
\xi_{t}=\exp \sum_{m=1}^{p-1} \sum_{J \in\{0 \ldots r\}^{m}} \beta^{J} \cdot B_{t}^{J} .
$$

$\operatorname{avec} \beta^{J}=\sum_{\sigma \in \sigma_{m}} C_{\lambda(\sigma)}\left[u_{j_{\sigma}-1_{(1)}},\left[u_{j_{\sigma}-1_{(2)}} \ldots u_{j_{\sigma}-1_{(m-1)}}, u_{j_{\sigma}-1_{(m)}}\right] \ldots\right]$. Or, ce $\beta^{J}$ est précisément le terme $m$ homogène de degré 1 en chacune des variables dans la série $H\left(u_{j_{1} \ldots u j_{m}}\right)$.

Ceci montre que:

Théorème 17. Les développements de $\xi_{t}$ obtenus par la méthode de Kunita (théorème 14) et les développements en série de Taylor stochastique (théorème 13) coüncident.

Remarque. Ce fait était clair a priori, mais on a voulu exhiber les calculs pour montrer la surprenante intéraction des formules algébriques et probabilistes. Remarquons que ces calculs permettent aussi de décrire le développement de $\xi_{t}$ dans le cas général (non nilpotent) à partir des intégrales multiples $\alpha_{t}^{T}$.

\section{(c) Le cas résoluble}

Dans le cas où l'algèbre de Lie est engendrée par $X_{0} \ldots X_{r}$ est résoluble, Kunita [11] obtient une représentation d'un type différent de la solution $\xi_{t}$ :

Théorème 18. Si Lie $\left(X_{0} \ldots X_{r}\right)$ est résoluble, soit $\left(Y_{1} \ldots Y_{n}\right)$ une base de l'algebre Lie $\left(X_{0}, \ldots, X_{r}\right)$ il existe $\left(N_{t}^{1} \ldots N_{t}^{n}\right) n$ semi-martingales continues telles que

$$
\xi_{t}=\exp N_{t}^{1} Y_{1} \ldots \exp N_{t}^{n} Y_{n}
$$

où les $N_{t}^{i}$ sont obtenues par répétition des opérations:

(1) Combinaisons linéaires et produits des $\left(B_{t}^{j}\right)$

(2) Intégration stochastique de Stratonovich contre $d B_{t}^{j}$

(3) Exponentiation. 
Ce théorème est simple; il suffit, par le théorème de Lie, de trigonaliser les ad $Y_{k}$ (cf. Kunita).

Le lien entre cette représentation et celle obtenue au théorème 12 est le lien usuel entre coordonnées canoniques de lère et de 2ème espèce. Pour passer de la forme du théorème 18 , à savoir coordonnées de seconde espèce à celle $\mathrm{du}$ théorème 12 , à savoir coordonnées de première espèce, il suffit d'appliquer une fois de plus la formule de Campbell-Hausdorff. Mais bien sûr, ceci n'est possible qu'avant un temps d'arrêt $T$, à savoir tant que $\left(N_{t}^{1} Y_{1}, \ldots, N_{t}^{n} Y_{n}\right)$ reste dans le domaine d'application de cette formule. Ce qui explique pourquoi le théorème 18 est valable pour tout $t$ et le théorème 12 seulement en temps petit. Plus précisément, avant ce temps $T$, on a:

$$
\xi_{t}=\exp H\left(N_{t}^{1} Y_{1}, \ldots, N_{t}^{n} Y_{n}\right) .
$$

$H\left(N_{t}^{1} Y_{1}, \ldots, N_{t}^{n} Y_{n}\right)$ peut s'écrire comme somme de Série de Taylor stochastique par application itérée de la formule d'Ito (pour transformer les produits en sommes d'intégrales itérées) et de généralisations évidentes du lemme suivant:

Lemme 6. La série $\sum_{0}^{\infty} \int_{0}^{t} \frac{\left(B_{s}^{1}\right)^{n}}{n !} \circ d B_{s}^{2}$ converge presque surement vers:

$$
\int_{0}^{t} e^{B_{s}^{1}} \circ d B_{s}^{2}
$$

ce qui permet de transformer les intégrales d'exponentielles en sommes intégrales itérées. Plutôt que de donner une preuve générale, on va s'attacher à l'exemple le plus simple, celui du groupe affine.

Soit $G=\left\{\left(\begin{array}{ll}1 & b \\ 0 & a\end{array}\right) a \in \mathbb{R}_{+}^{*}, b \in \mathbb{R}\right\}$ le groupe affine et $\mathscr{G}=\left\{\left(\begin{array}{ll}0 & x \\ 0 & y\end{array}\right),(x, y) \in \mathbb{R}^{2}\right\}$ son algèbre de Lie.

Choisissons la base $\left(u_{1}, u_{2}\right)$ de $\mathscr{G}$ donnée par $u_{1}=\left(\begin{array}{rr}0 & 0 \\ 0 & -1\end{array}\right) u_{2}=\left(\begin{array}{ll}0 & 1 \\ 0 & 0\end{array}\right)$ de telle sorte que $\left[u_{1}, u_{2}\right]=u_{2}$.

Considérons alors les champs de vecteurs $X_{1}, X_{2}$ sur $G$, invariants à droite, associés à $u_{1}, u_{2}$ et $\xi_{t}=\left(\begin{array}{ll}1 & b_{t} \\ 0 & a_{t}\end{array}\right)$ la solution sur $G$ de l'équation:

$$
\begin{aligned}
\xi_{0} & =e \\
d \xi_{t} & =\sum_{i=1}^{2} X_{i}\left(\xi_{t}\right) \circ d B_{t} .
\end{aligned}
$$

Cette équation se résoud par quadrature, et on obtient:

$$
\begin{aligned}
& a_{t}=e^{-B_{t}^{1}} \\
& b_{t}=\int_{0}^{t} e^{-B_{s}^{1}} d B_{s}^{2} .
\end{aligned}
$$


Ce qui donne très simplement la représentation de $\xi_{t}$ en coordonnées de secondes espèces, valable pour tout $t$ :

$$
\xi_{t}=\exp \left(B_{t}^{1} u_{1}\right) \exp \left(\int_{0}^{t} e^{-B_{s}^{1} \circ} d B_{s}^{2}\right) u_{2}
$$

ce qui est conforme au résultat de Kunita.

D'autre part, l'application exponentielle est ici très simple sur $\mathscr{G}$; on a:

$$
\exp \left(\begin{array}{ll}
0 & x \\
0 & y
\end{array}\right)=\left(\begin{array}{cc}
1 & x \frac{e^{y}-1}{y} \\
0 & e^{y}
\end{array}\right) \quad \text { avec } \frac{e^{0}-1}{0}=1
$$

C'est un difféomorphisme de $\mathscr{G}$ sur $G$ et son inverse est donné par:

$$
\begin{aligned}
\log \left(\begin{array}{ll}
1 & b \\
0 & a
\end{array}\right) & =\left(\begin{array}{cc}
0 & \frac{\log a}{a-1} \\
0 & \log a
\end{array}\right) \\
& =(-\log a) u_{1}+\left(\frac{\log a}{a-1} \cdot b\right) u_{2}
\end{aligned}
$$

D'où

$$
\xi_{t}=\exp \left[B_{t}^{1} u_{1}+\left(\int_{0}^{t} e^{-B_{s}^{1}} \circ d B_{s}^{2}\right) \frac{-B_{t}^{1}}{e^{-B_{s}^{1}}-1} u_{2}\right]
$$

Soit $T$ le temps d'arrêt $T=\inf \left(T,\left|B_{t}^{1}\right| \geqq 2 \pi\right)$. Sur [0, $T$ [ le développement suivant est absolument convergent.

$$
\frac{B_{t}^{1}}{e^{-B_{t}^{1}}-1}=1-\frac{B_{t}^{1}}{2}+\sum_{i}^{\infty}(-1)^{n-1} \frac{b_{n}}{(2 n) !}\left(B_{t}^{1}\right)^{2 n}
$$

d'où, par le lemme 6 , sur $[0, T[$ le produit des séries de terme général $\int_{0}^{t} \frac{B_{s}^{1} n}{n !} \circ d B_{s}^{2}$ et $(-1)^{n-1} \frac{b_{n}}{(2 n) !}\left(B_{t}^{1}\right)^{2 n}$ converge vers $\left(\int_{0}^{t} e^{-B_{s}^{1}} \circ d B_{s}^{2}\right)\left(\frac{B_{t}^{1}}{e^{-B_{t}^{1}-1}}\right)$. Ainsi, ce dernier produit s'écrit comme somme presque sure sur $[0, T[$ d'une série dont le terme général est une combinaison linéaire de termes de la forme:

$$
\left(B_{t}^{1}\right)^{2 m} \int_{0}^{t}\left(-B_{t}^{1}\right)^{n} \circ d B_{s}^{2}
$$

Chacun de ces termes peut, à son tour, être écrit comme combinaison linéaire d'intégrales itérées de Stratonovich d'ordre $2 m+n+1$. 
Remarquons que le terme de plus bas degré de cette série est égal à $B_{t}^{2}$. On a ainsi montré directement (i.e., sans faire appel au théorème 12) qu'il existe un temps d'arrêt $T$ et des réels $\lambda_{J}$, tels que sur $[0, T[$ on ait:

$$
\xi_{t}=\exp \left(B_{t}^{1} u_{1}+B_{t}^{2} u_{2}+\sum_{m=2} \sum_{J \in\{1,2\}^{m}} \lambda_{J} B_{t}^{J} u_{2}\right)
$$

qui est bien un développement du type de celui du théorème 12, car les seuls crochets des $u_{1}, u_{2}$ non nuls sont égaux ici à $u_{2}$.

Remarquons qu'ici le temps d'arrêt $T$ est explicitement décrit; en fait, on peut aussi l'interpréter comme le temps de première sortie de $\xi_{t}$ hors de l'ouvert $\exp V$, où $V=\{x \in \mathscr{G} /$ le rayon spectral de ad $x$ est $<2 \pi\}$. En effet, si $x \in \mathscr{G}$ s'écrit $x_{1} u_{1}+x_{2} u_{2}$, le rayon spectral de ad $x$ est égal à $\left|x_{1}\right|$. Ainsi, $\zeta_{t}=\log \xi_{t} \notin V \Leftrightarrow\left|B_{t}^{1}\right|$ $\geqq 2 \pi$. C.Q.F.D.

Remarque. Il reste à prouver le lemme 6. Pour cela, on a, pour tout $x$ de $\mathbb{R}$, par la formule de Taylor usuelle:

$$
\left|e^{x}-\sum_{0}^{n-1} \frac{x^{k}}{k !}\right| \leqq \frac{|x|^{n}}{n !} e^{|x|}
$$

D'où, si $s \leqq t$ :

$$
E\left(\left(e^{B_{s}^{1}}-\sum_{0}^{n-1} \frac{\left(B_{s}^{1}\right)^{k}}{k !}\right)\right) \leqq \frac{1}{n !^{2}} E\left(\left|B_{s}^{1}\right|^{2 n} e^{2\left|B_{s}^{1}\right|}\right)
$$

Un calcul très simple montre que:

$$
E\left(\left|B_{s}^{1}\right|^{2 n} e^{2\left|B_{s}^{1}\right|}\right) \leqq 2 e^{2 t}(16 t)^{n}\left(t^{n}+\frac{2 n !}{2^{4 n} n !}\right) \quad \text { pour tout } s \leqq t .
$$

D'où l'on obtient:

$$
E\left(\left(\int_{0}^{t} e^{B_{s}^{1}} \circ d B_{s}^{2}-\sum_{0}^{n-1} \int_{0}^{t} \frac{\left(B_{s}^{1}\right)^{k}}{k !} \circ d B_{s}^{2}\right)^{2}\right) \leqq 2 t e^{2 t} \frac{(16 t)^{n}}{n !}\left(\frac{t^{n}}{n !}+\frac{2 n !}{2^{4 n} n !^{2}}\right)
$$

et, par l'inégalité de Tchebichev, en posant $\lambda_{n}=\sqrt{\frac{(16 t)^{n}}{n !}}$ :

$$
P\left(\left|\int_{0}^{t} e^{B_{s}^{1}} \circ d B_{s}^{2}-\sum_{0}^{n-1} \int_{0}^{t} \frac{B_{s}^{1^{k}}}{k !} \circ d B_{s}^{2}\right|>\lambda_{n}\right) \leqq 2 t e^{2 t}\left(\frac{t^{n}}{n !}+\frac{2 n !}{2^{4 n} n !^{2}}\right)
$$

Or, la série de droite est convergente par la formule de Stirling, d'où, par le lemme de Borel-Cantelli, le résultat est démontré. 


\section{Application aux diffusions invariantes par l'action d'un groupe, lien avec les résultats de Yamato-Fliess-Kunita}

Soit $M$ une variété $C^{\infty}$ connexe et $G$ un groupe de Lie qui agit à gauche sur $M$; l'action $\phi: G \times M \rightarrow M,(g, m) \rightarrow \phi(g, m)$ définit un morphisme $\phi^{+}$de l'algèbre de Lie $\mathscr{R}$ des champs de vecteurs sur $G$ invariants à droite, dans l'algèbre de Lie des champs de vecteurs $C^{\infty}$ sur $M$ par.

$$
\text { Si } Y \in \mathscr{S} \quad \phi^{+}(Y)(x)=T_{e} \phi(\cdot, x) \cdot Y(e) \quad \text { pour } x \in M .
$$

Considérons sur $M r+1$ champs $X_{i}=\phi^{+}\left(Y_{i}\right)$ pour $Y_{i} \in \mathscr{R}$, et l'équation stochastique associée aux $X_{i}$ :

$$
\begin{aligned}
\xi_{0} & =x \\
d \xi_{t} & =\sum_{i=0}^{r} X_{i}\left(\xi_{t}\right) \circ d B_{t}^{i} .
\end{aligned}
$$

Théorème 19. (1) $\xi_{t}$ a un temps de vie infini.

(2) Il existe un temps d'arrêt $T$ presque surement strictement positif, tel que: sur $t<T$, on ait:

$$
\xi_{t}=\exp \left(\sum_{m=1}^{\infty} \sum_{\|\boldsymbol{J}\|=m} \beta_{J} B_{t}^{J}\right)(x)
$$

où $\beta_{J}$ est le terme $m$ homogène de degré 1 en chacune des variables dans la série de Campbell-Hausdorff $H\left(X_{j_{1}}, \ldots, X_{j_{m}}\right)$ et où l'exponentielle est prise ici au sens de flot au temps 1 d'un champ de vecteur.

Preuve. Ce théorème est évident. Il suffit de considérer sur $G$ la solution de l'équation

$$
\begin{aligned}
d \xi_{t} & =\sum_{i=0}^{r} Y_{i}\left(\xi_{t}\right) \circ d B_{t}^{i} \\
\xi_{0} & =e
\end{aligned}
$$

$\xi_{t}$ a un temps de vie infini et la formule d'Ito montre que $\phi\left(\xi_{t}, x\right)$ est solution sur $M$ de l'équation associée aux champs $\phi^{+}\left(Y_{i}\right)=X_{i}$ à savoir de l'équation (1). Ceci montre que $\xi_{t}=\phi\left(\zeta_{t}, x\right)$ d'où le 1). Mais si $Y$ est un champ invariant à droite $\operatorname{sur} M$, on $\mathbf{a}$ :

$$
\phi(\exp Y(e), x)=\left[\exp \phi^{+}(Y)\right](x)
$$

Il suffit donc d'appliquer le théorème 12 pour obtenir le 2).

On a énoncé le théorème essentiellement pour le corollaire suivant:

Corollaire 2. Soient $M$ une variété $C^{\infty}$ connexe et $X_{0} \ldots X_{r} \in \mathscr{X}(M)$ tels que

(1) Les $X_{i}$ soient des champs complets,

(2) Lie $\left(X_{0} \ldots X_{r}\right)$ est de dimension finie.

Alors, les conclusions du théorème 1 sont vraies. 
Preuve. Le théorème de Palais [15] montre que sous les hypothèses (1) et (2), il existe un unique groupe de Lie connexe $G$ inclus dans le groupe des difféomorphismes $C^{\infty}$ de $M$ qui agit à gauche sur $M$ par $\phi G \times M \rightarrow M(g, x) \mapsto g(x)$ et tel que $\phi^{+}$soit un isomorphisme de $\mathscr{G}$ sur Lie $\left(X_{0} \ldots X_{r}\right)$. G est le groupe engendré par les $\exp t X$ pour $X \in \operatorname{Lie}\left(X_{0} \ldots X_{r}\right)$ muni d'une topologie connexe par arcs plus fine en général que la topologie compacte ouverte. Considérons alors les champs $Y_{i}=\phi^{+-1}\left(X_{i}\right)$ invariants à droite sur $G$; on est dans la situation du théorème précédent.

Ce théorème montre donc que, sous les hypothèses (1) et (2) du corollaire, on a considérablement amélioré les résultats du paragraphe 3. Le flot stochastique défini par l'équation (1) est, en temps petit, le flot ordinaire au temps 1 du champ de vecteur aléatoire $X_{t}=\sum_{m=1}^{\infty} \sum_{\|J\|=m} \beta_{J} B_{t}^{J}$, où la série converge ici sans hypothèse d'analyticité des $X_{i}$ et où les coefficients des $B_{t}^{J}$ sont parfaitement explicites.

Il faut constater ici que l'on a relevé une équation $C^{\infty}$ en une équation analytique au travers d'une application $C^{\infty}$. Ceci est rendu possible par la condition très forte (2). Ceci suscite une question ouverte: la condition (2) est-elle nécessaire pour que les conclusions du théorème soient vérifiées? En particulier, on retrouve ici les résultats de Yamato [18] et Fliess et Normand-Cyrot [7]

Corollaire 3. $\mathrm{Si}$ (1) Lie $\left(X_{0}, \ldots, X_{r}\right)$ est p-nilpotente et

(2) Les champs $X_{i}$ sont complets,

alors pour tout $t: \xi_{t}(x)=\exp \left(\sum_{m=1}^{p-1} \sum_{\|J\|=m} \beta_{J} B_{t}^{J}\right)(x)$, et donc il existe une fonction $F C^{\infty}$, telle que:

$$
\xi_{t}(x)=F\left(x,\left(B_{t}^{J}\right)\right)
$$

Il suffit de nouveau d'appliquer le théorème 13 .

Remarquons ici que ce résultat précise celui de Yamato dans le sens où il montre qu'il s'agit d'un développement de Taylor stochastique et qu'il explicite entièrement la fonction $F$. De plus, notre résultat est valable sur toute variété et, surtout, la méthode des développements de Taylor stochastiques se prète à la généralisation au cas non nilpotent.

Enfin, remarquons qu'il est nécessaire de supposer les champs complets pour obtenir un résultat valable pour tout $t$ (Yamato les suppose même lipschitziens), cela se comprend ici aisément, car si les $X_{i}$ ne sont pas complets, rien n'assure que le flot de $\Sigma \beta_{J} B_{t}^{J}$ soit défini au temps 1 pour tout $t$.

\section{(2) Extension aux champs non complets}

En fait, si l'on n'est intéressé que par le développement en temps petit, qui est une question locale, il est un peu ridicule d'utiliser le théorème de Palais, dont la caractéristique (et la difficulté) est d'être global. Il suffit d'utiliser sa version locale qui est la réciproque du $2^{\text {nd }}$ théorème de Lie. On montre ainsi le résultat: 
Théorème 20. Soient $X_{0}, \ldots, X_{r} \in \mathscr{X}(M)$, tels que:

(1) Lie $\left(X_{0}, \ldots, X_{r}\right)$ soit de dimension finie.

(2) $\operatorname{dim}\left(\right.$ Lie $\left(X_{0} \ldots X_{r}\right)(x)$ est constant au voisinage de $x_{0}$; alors, il existe un temps d'arrêt $T$ presque surement strictement positif, tel que, sur $t<T$, on ait:

$$
\xi_{t}(x)=\exp \left(\sum_{m=1}^{\infty} \sum_{|J|=m} \beta_{J} B_{t}^{J}\right)(x) .
$$

Preuve. Par la réciproque du $2^{\text {nd }}$ théorème de Lie, on sait que, sue la feuille $\Gamma$, associée à la distribution Lie $\left(X_{0} \ldots X_{r}\right)(x)$, par le théorème de Frobénius, il existe, sur un voisinage $U$ de $x_{0}$ inclus dans $\Gamma$, une structure de groupe de Lie local, tel que les $X_{i}$ soient des champs invariants à droite pour cette structure de groupe. Il suffit alors d'appliquer directement les résultats du paragraphe 4(a) en utilisant la remarque faite alors qu'ils n'utilisent que la structure de groupe local de $G$.

Ce théorème permet d'omettre l'hypothèse de complétude des champs $X_{i}$.

\section{Le mouvement Brownien sur une variété Riémannienne}

Soit $(M, g)$ une variété riemannienne $C^{\infty}$ de dimension $n$. Soit $m \in M$, l'application exponentielle en $m \exp _{m}$ définit un difféomorphisme d'un voisinage $V$ de 0 dans $T_{m} M$ sur un voisinage $W$ de $m$ dans $M$.

Fixons un repère orthonormé $\left(u_{i}\right)$ de $T_{m} M$; on définit ainsi une carte normale au voisinage de $m$ par:

$$
\left(x_{1} \ldots x_{m}\right) \rightarrow \exp _{m}\left(\sum_{i}^{n} x_{i} u_{i}\right)
$$

Soit $\Delta$ l'opérateur de Laplace-Beltrami sur $M$ et $\xi_{t}$ le mouvement brownien, à savoir la diffusion de générateur $\Delta$ issue de $m$. Les techniques précédentes de développement de Taylor stochastique permettent de donner une description universelle de $\xi_{t}$ en carte normale.

Théorème 21. (1) Soit $T_{W}=\inf \left(t, \xi_{t} \notin W\right)$ alors, sur $t<T_{W}$, on $a$

$$
\xi_{t}=\exp _{m} \sum_{i=1}^{n} x_{t}^{i} u_{i}
$$

avec

$$
x_{t}^{i}=\sum_{k=1}^{N} \sum_{\|J\|=k} P_{J}^{i} B_{t}^{J}+t^{N+1 / 2} R_{N}^{i}(t)
$$

où le reste $R_{N}^{i}(t)$ est borné en probabilité lorsque $t \rightarrow 0$, et où les $P_{J}^{i}$ sont des polynomes universels en les dérivées covariantes successives, en $m$, du tenseur de courbure $R$. 
Précisément, les premiers termes sont donnés par:

$$
x_{t}^{i}=B_{t}^{i}+t^{3 / 2} R_{2}^{i}(t)
$$

Ainsi, le mouvement brownien sur $M$ est en temps petit égal à un mouvement brownien euclidien au troisième ordre près. Le terme du second ordre s'annule, la correction due à la courbure n'apparaît qu'au troisième ordre.

(2) Si $(M, g)$ est une variété riemannienne analytique, alors il existe un temps d'arrêt $T$ presque surement strictement positif, tel que, sur $t<T$, on ait:

$$
x_{t}^{i}=\sum_{k=1}^{\infty} \sum_{\|J\|=k} P_{J}^{i} B_{t}^{J}
$$

Le mouvement brownien sur $M$ est ainsi, en coordonnées normales, une fonction déterministe universelle des dérivées covariantes de la courbure et des intégrales stochastiques itérées.

Preuve. Dans n'importe quelle carte, l'opérateur de Laplace-Beltrami $\Delta$ s'écrit:

$$
\Delta f=\frac{1}{2} \vartheta^{-1} \sum_{i, j} \frac{\partial\left(\vartheta g^{i j} \frac{\partial f}{\partial x_{j}}\right)}{\partial x_{i}}
$$

où $\vartheta=\sqrt{\operatorname{det} g_{i j}}$

$$
\Delta f=\frac{1}{2} \Sigma g^{i j} \frac{\partial f}{\partial x_{i} \partial x_{j}}+\sum_{j} \frac{1}{2}\left(\sum_{i} \frac{\partial g^{i j}}{\partial x_{i}}+\left(\vartheta^{-1} \frac{\partial \vartheta}{\partial x_{i}}\right) g^{i j}\right) \frac{\partial f}{\partial x_{j}} .
$$

Si $X=\left(X_{i}^{j}\right)$ désigne une racine carrée $C^{\infty}$ quelconque de $g^{-1}$, à savoir si $X X^{*}$ $=\mathrm{g}^{-1}$ (ou encore, de façon équivalente, si les champs de vecteurs colonnes de $X: X_{i}=\left(X_{i}^{j}\right)_{j \in\{1 \ldots n\}}$ forment un répère orthonormé pour $g$ ), on a:

$$
\Delta=\frac{1}{2} \sum_{i=1}^{n} X_{i}^{2}+X_{0}
$$

où le drift $X_{0}$ est donné par:

$$
X_{0}^{j}=\frac{1}{2} \sum_{i}\left(\frac{\partial g^{i j}}{\partial x_{i}}+\left(\vartheta^{-1} \frac{\partial \vartheta}{\partial x_{i}}\right) g^{i j}\right)+\sum_{k} X_{i}^{k} \frac{\partial X_{i}^{j}}{\partial x_{k}} .
$$

L'intérêt d'un système de carte normal est le suivant:

Théorème 22. Second théorème d'Elie Cartan (cf. Berger [3]). Les coefficients de Taylor de $\left(g_{i j}\right)$ au voisinage de $x=0 \in T_{m} M$ sont des polynomes universels en 
les dérivées covariantes successives du tenseur de courbure au point m En particulier:

$$
\left.\begin{array}{l}
g_{i j}(0)=\delta_{i j} \\
g_{i j}(0)=0 \\
g_{i j}()=-R_{i j k l}(m) .
\end{array}\right\}
$$

Choisissons alors, parmi toutes les racines carrées possibles de $\left(g^{i j}\right)$ la racine symétrique donnée par

où l'on a noté:

$$
X=\Sigma c_{n}\left(g^{-1}-I\right)^{n}
$$

$$
\sqrt{1-z}=\sum_{n=0}^{\infty} c_{n} z^{n}
$$

Cette série converge normalement pour $\left\|g^{-1}-I\right\|<1$, donc sur un voisinage de $m$.

Les coefficients de Taylor en $m$ des champs $X^{i}$, colonnes de la matrice $X$ sont alors des polynomes universels en ceux de $g^{-1}$ et donc en ceux de $g$. Ce sont donc des polynomes universels en les dérivées covariantes de la courbure.

Considérons enfin le drift:

$$
X_{0}=\frac{1}{2}\left[\sum_{i} \frac{\partial g^{i j}}{\partial x_{i}}+\left(\vartheta^{-1} \frac{\partial \vartheta}{\partial x^{i}}\right) g^{i j}+\Sigma X_{i}^{k} \frac{\partial X_{i}^{j}}{\partial_{x} k}\right] .
$$

Ici $\vartheta=\sqrt{\operatorname{det} g_{i j}}$, la dérivée $n^{\text {eme }}$ de $\vartheta$ en 0 est un polynome universel en les variables $\left[\operatorname{det} g_{i j}(0)\right]^{-k / 2}$ et $\left(\operatorname{det} g_{i j}\right)^{(l)}(0)$. Or ici, det $g_{i j}(0)=1$ et la dérivée lème en 0 de det $g_{i j}$ est un polynome universel en les dérivées de $g_{i j}$ en 0 . Ceci montre (en utilisant la remarque faite précédemment sur les coefficients de Taylor des champs $X_{i}$ ) que les coefficients de Taylor de $X_{0}$ en 0 sont des polynomes universels en les dérivées de $g_{i j}$ en 0 et donc en les dérivées covariantes successives de la courbure en $m$.

Si l'on considère alors la solution sur $W$ de l'équation

$$
\begin{aligned}
d \xi_{t} & =\sum_{i=0}^{r} X_{i}\left(\xi_{t}\right) \circ d B_{t}^{i} \\
\xi_{0} & =m .
\end{aligned}
$$

$\xi_{t}$ est bien la diffusion sur $W$, issue de $m$, de générateur $\frac{1}{2} \sum_{i}^{r} X_{i}^{2}+X_{0}=\Delta$ à savoir le mouvement brownien.

Il suffit alors d'appliquer les théorèmes 6 et $9 \mathrm{du}$ paragraphe 1 , pour conclure, d'une part, à l'existence des développements 1) et 2) pour les coordonnées normales de $\xi_{t}$, et, d'autre part, à l'identification des $P_{J}^{i}$ comme polynomes universels 
en les coefficients de Taylor en $m$ des $X_{i}$ et donc par ce que l'on vient de voir en les dérivées covariantes successives de la courbure prises au point $m$.

Le calcul des premiers termes se fait en utilisant le système $\mathscr{S}_{m}$, qui définit la série de Taylor stochastique, et les relations (*). On obtient ainsi que:

$$
g_{1}(t)=\sum_{i=1}^{r} X_{i}(m) B_{t}^{i}=\sum_{i=1}^{r} u_{i} B_{t}^{i}
$$

et que $g_{2}(t)=0$, car $X_{0}(m)=0$ et $\frac{\partial X_{i}^{j}}{\partial x_{k}}(m)=0$, ce qui termine la preuve du théorème.

\section{Appendice 1: Preuve du théorème 1}

Preuve. La preuve se fait par récurrence sur $|J|$. Si $|J|=1$, le théorème est évident avec $a(J)=1$.

Supposons le théorème vérifié pour tout multi-indice $K$ tel que $|K|<m$. Soit alors $J=\left(j_{1} \ldots j_{m}\right) \in\{0 \ldots r\}^{m}$. Notons $J_{k}=\left(j_{1} \ldots j_{m-k}\right)$.

$$
\begin{aligned}
\text { Si } j_{m} \neq 0 \text { on } a: E\left(I_{t}^{J^{2}}\right) & =\int_{0}^{t} E\left(I_{s}^{J^{2}}\right) d s=\frac{1}{a\left(J_{1}\right)} \int_{0}^{t} t^{\| J^{\|}} d s \\
E\left(I_{t}^{J^{2}}\right) & =\frac{t^{\|J\|}}{a\left(J_{1}\right)\left\|J_{1}\right\|+1} .
\end{aligned}
$$

Or ici $\|J\|=\left\|J_{1}\right\|+1$.

D'où $a(J)=a\left(J_{1}\right)\left(\left\|J_{1}\right\|+1\right)=a\left(J_{1}\right)\|J\|=\|J\| ! \prod_{i}^{k} \frac{\left(p_{i}\right) !^{2}}{\left(2 p_{i}\right) !}$ par l'hypothèse de récurrence; en effet, les $\left(p_{i}\right)$ associés à $J_{1}$ et à $J$ sont identiques.

Si $j_{m}=0$ :

a) Si tous les $j_{i}$ sont nuls, alors $I_{t}^{J}=\frac{t^{m}}{m !}$ d'où $a(J)=m !^{2}$. Ici $\|J\|=2 m, k=1$ et $p_{1}=m$; on a bien $a(J)=\|J\| ! \frac{p_{1} !^{2}}{\left(2 p_{1}\right) !}=m !^{2}$.

(b) Si l'un des $j_{i}$ est non nul: soit $p=\inf \left(i, j_{m-i} \neq 0\right), 1 \leqq p \leqq m-1$.

Considérons alors le multi-indice $J_{p}=\left(j_{1} \ldots j_{m-p}\right)$. Alors $k(J)=k\left(J_{p}\right)+1$ et $p_{i}(J)=p_{i}\left(J_{p}\right)$ pour $i \leqq k\left(J_{p}\right)$ et $p_{i}(J)=p$ pour $i=k(J)$ et $\|J\|=\left\|J_{p}\right\|+2 p$.

Montrons alors que: $a(J)=a\left(J_{p}\right) \frac{p !}{(2 p) !}\left(\left\|J_{p}\right\|+1\right) \ldots\left(\left\|J_{p}\right\|+2 p\right)(*)$, ce qui terminera la preuve du théorème, puisque, par l'hypothèse de récurrence, $a\left(J_{p}\right)$ $=\left\|J_{p}\right\| ! \prod_{i}^{k\left(J_{p}\right)} \frac{p_{i} !^{2}}{\left(2 p_{i}\right) !}$.

Pour cela, on va démontrer le lemme suivant:

Lemme 1. Pour $k \leqq p, E\left(I_{t}^{J_{k}} I_{t}^{J_{p}^{p}}\right)=\underset{0<t_{1}<\ldots<t_{p-k}<t}{\int \ldots \int} E\left(I_{t_{k}}^{J_{k}^{2}}\right) d t_{1} \ldots d t_{p-k}$. 
La preuve se fait par récurrence descendante sur $k$. Le lemme est évident

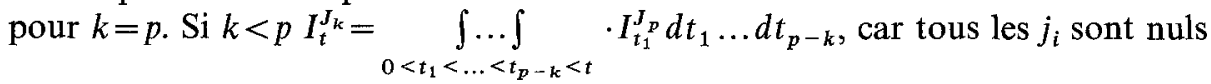
pour $i>m-p$, d'où $I_{t}^{J_{k}}=\int_{0}^{t} I_{s}^{J_{k+1}} d s$. On a alors, par la formule d'Ito:

$$
I_{t}^{J_{k}} I_{t}^{J_{p}}=\int_{0}^{t} I_{s}^{J_{k}} d I_{s}^{J_{p}}+\int_{0}^{t} I_{s}^{J_{p}} d I_{s}^{J_{k}}
$$

car $I^{J_{k}}$ est à variations bornées. Or $I_{s}^{J_{p}}$ est une martingale car $j_{m-p} \neq 0$, d'où

$$
E\left(\int_{0}^{t} I_{s}^{J_{k}} d I_{s}^{J_{p}}\right)=0
$$

et donc

$$
\begin{aligned}
E\left(I_{t}^{J_{k}} I_{t}^{J_{p}}\right) & =E \int_{0}^{t} I_{s}^{J_{p}} d I_{s}^{J_{k}}=E\left(\int_{0}^{t} I_{s}^{J_{p}} I_{s}^{k+1} d s\right) \\
& =\int_{0}^{t} E\left(I_{s}^{J_{p}} I_{s}^{J_{k+1}}\right) d s,
\end{aligned}
$$

et le lemme est démontré par récurrence sur $k$.

Du lemme 1, on tire:

Lemme 2. (1) Pour $k \leqq p$,

$$
E\left(I_{k_{t}}^{J} I_{t}^{J_{p-1}}\right)=(p-k+1) \underset{0<t_{1}<\ldots<t_{p-k+1}<t}{\int \ldots \int} E\left(\left(I_{t_{1}}^{J_{p}}\right)^{2}\right) d t_{1} \ldots d t_{p-k+1},
$$

(2) Pour $k \leqq p$ et $l \leqq p$ :

$$
E\left(I_{t}^{J_{k}} I_{t}^{J_{t}}\right)=C_{2 p-(k+l)}^{p-k} \underset{0<t_{1}<\ldots<t_{2 p-(k+l)<t}}{\int \ldots \int} E\left(\left(I_{t_{1}}^{\left.J_{p}\right)^{2}}\right) d t_{1} \ldots d t_{2 p-(k+l)} .\right.
$$

Preuve $d u(1)$. On a $I_{t}^{J_{p-1}}=\int_{0}^{t} I_{s}^{J_{p}} d s$ et par la formule d'Ito, si $k<p$ :

$$
\begin{aligned}
I_{t}^{J_{k}} I_{t}^{J_{p-1}} & =\int_{0}^{t} I_{s}^{J_{k}} d I_{s}^{J_{p-1}}+\int_{0}^{t} I_{s}^{J_{p-1}} d I_{s}^{J_{k}} \\
& =\int_{0}^{t} I_{s}^{J_{k}} I_{s}^{J_{p}} d s+\int_{0}^{t} I_{s}^{J_{p-1}} I_{s}^{J_{k+1}} d s
\end{aligned}
$$


La preuve se fait de nouveau par récurrence descendante sur $k$ : si $k=p$ :

$$
\begin{aligned}
I_{t}^{J_{k}} I_{t}^{J_{p-1}} & =\int_{0}^{t} I_{s}^{J_{p}} d I_{s}^{J_{p-1}}+\int_{0}^{t} I_{s}^{J_{p-1}} d I^{J_{p}} \\
& =\int_{0}^{t}\left(I_{s}^{J_{p}}\right)^{2} d s+\int_{0}^{t} I_{s}^{J_{p-1}} d I_{s}^{J_{p}}
\end{aligned}
$$

$I^{J_{p}}$ étant une martingale, on a $E\left(I_{t}^{J_{k}} I_{t}^{J_{p-1}}\right)=\int_{0}^{t} E\left(I_{s}^{J_{p}}\right)^{2} d s$, et le lemme est vérifié dans ce cas.

La formule $(* *)$ montre que si $k<p$ :

$$
E\left(I_{t}^{J_{k}} I_{t}^{J_{p-1}}\right)=\int_{0}^{t} E\left(I_{s}^{J_{k}} I_{s}^{J_{p}}\right) d s+\int_{0}^{t} E\left(I_{s}^{J_{p-1}} I_{s}^{J_{k+1}}\right) d s .
$$

Donc par le lemme 1

$$
E\left(I_{t}^{J_{k}} I_{t}^{J_{p-1}}\right)=\underset{0<t_{1}<\ldots<t_{p-k+1}<t}{\int \ldots \int_{t_{1}}} E\left(I_{t_{1}^{2}}^{J_{1}}\right) d t_{1} \ldots d t_{p-k+1}+\int_{0}^{t} E\left(I_{s}^{J_{p-1}} I_{s}^{J_{k+1}}\right) d s .
$$

Le dernier terme de cette égalité est par l'hypothèse de récurrence égal à ( $p$ $-k) \underset{0<t_{1}<\ldots<t_{p-k+1}<t}{\int \ldots \int} E\left(I_{t_{1}^{\prime}}^{J_{1}^{2}}\right) d t_{1} \ldots d t_{p-k+1}$, d'où le (1) du lemme est démontré.

Preuve $d u$ (2). De nouveau, ce lemme se démontre par récurrence descendante. Ici, sur $k+l$ : Si $k+l=2 p$ le résultat est évident. Si $k+l=2 p-1$ c'est une conséquence du lemme 2 (1). Pour $k+l \leqq 2 p-2$, on a, de nouveau, par la formule d'Ito: (puisque $k \leqq p-1, l \leqq p-1$ )

$$
E\left(I_{t}^{J_{k}} I_{t}^{J_{l}}\right)=\int_{0}^{t} E\left(I_{s}^{J_{k}} I_{s}^{J_{l+1}}\right) d s+\int_{0}^{t} E\left(I_{s}^{J_{l}} I_{s}^{J_{k+1}}\right) d s
$$

Par l'hypothèse de récurrence, le premier terme de cette égalité s'écrit

$$
C_{2 p-(k+l)-1}^{p-k} \int_{0<t_{1}<\ldots<t_{2 p-(k+l)}<t} E\left(I_{t_{1}^{p}}^{\left.J_{p}^{2}\right)} d t_{1} \ldots d t_{2 p-(k+l)} .\right.
$$

De même, par l'hypothèse de récurrence, le second terme s'écrit

$$
C_{2 p-(k+l)-1}^{p-(k+1)} \underset{0<t_{1}<\ldots<t_{2 p-(k+t)}<t}{\int \ldots \int} E\left(I_{t_{1}^{p}}^{\left.J_{p}^{2}\right)} d t_{1} \ldots d t_{2 p-(k+1)} .\right.
$$

Or $C_{2 p-(k+l)-1}^{p-k}+C_{2 p-(k+l)-1}^{p-(k+1)}=C_{2 p-(k+l)}^{p-k}$ et le (2) du lemme est démontré. En particulier, le lemme 2 montre que si $k \leqq p$

$$
E\left(\left(I_{t}^{J_{k}}\right)^{2}\right)=C_{2(p-k)}^{p-k} \underset{0<t_{1}<\ldots<t_{2(p-k)}<t}{\int \ldots \int} E\left(\left(I_{t}^{\left.J^{p}\right)^{2}}\right) d t_{1} \ldots d t_{2(p-k)} .\right.
$$


En particulier pour $k=0$ :

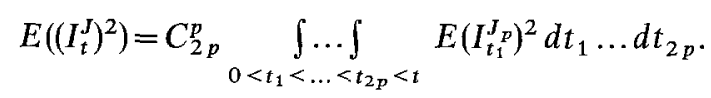

$\operatorname{Or} E\left(I_{t}^{J_{p}^{2}}\right)=\frac{t_{1}^{\left\|J_{p}\right\|}}{a\left(J_{p}\right)}$ d'où

$$
E\left(I_{t}^{J^{2}}\right)=C_{2 p}^{p} \frac{t^{\left\|J_{p}\right\|}+2}{\left(\left\|J_{p}\right\|+1\right) \ldots\left(\left\|J_{p}\right\|+2 p\right)} \frac{1}{a\left(J_{p}\right)}=\frac{t^{\|J\|}}{a(J)} \operatorname{car}\|J\|=\left\|J_{p}\right\|+2 p
$$

$\operatorname{avec} a(J)=a\left(J_{p}\right)\left(\left\|J_{p}\right\|+1\right) \ldots\left(\left\|J_{p}\right\|+2 p\right) \frac{1}{C_{2 p}^{p}}$

$$
a(J)=a\left(J_{p}\right)\left(\left\|J_{p}\right\|+1\right) \ldots\left(\left\|J_{p}\right\|+2 p\right) \frac{p !^{2}}{(2 p)} !
$$

Ceci est la relation $(*)$ cherchée et donc achève la preuve du théorème.

\section{Appendice 2: Preuve du lemme 4}

Ce lemme est évident pour $k=1$, en effet $g_{1}(t)=\sum_{i=1}^{r} u_{i} B_{t}^{i}$ et $C_{i}^{1}\left(g_{1}(t)\right)$ est le coefficient de $x$ dans la série de Taylor de $\sigma_{i}\left(x g_{1}\right)$, à savoir $\frac{1}{2}\left[g_{1}, u_{i}\right]$ ce qui est bien égal à $\sum_{\|J\|=1} \beta_{J \cup\{i\}} B_{t}^{J}=\sum_{j=1}^{r} \beta_{j, i} B_{t}^{j}$, ou encore $\frac{1}{2} \sum_{j=1}^{r}\left[u_{j}, u_{i}\right] B_{t}^{j}$.

Supposons le lemme vérifié pour $k \leqq m-1$; on a alors, de façon claire:

$$
g_{m}(t)=\sum_{i=1}^{r} \sum_{\|J\|=m-1} \beta_{J \cup\{1\}} \int_{0}^{t} B_{s}^{J} \circ d B_{s}^{i}+\sum_{\|J\|=m-2} \beta_{J \cup\{0\}} \int_{0}^{t} B_{s}^{J} \circ d B_{s}^{0} .
$$

d'où: $g_{m}(t)=\sum_{\|K\|=m} \beta_{K} B_{t}^{K}$ et le a) du lemme est vérifié à l'ordre $m$.

La difficulté est de prouver que le b) l'est:

Par définition $C_{i}^{m}\left(g_{1}(t) \ldots g_{m}(t)\right)$ est le coefficient de $x^{m}$ dans la série de Taylor en 0 de $\sigma_{i}\left(\sum_{k=1}^{m} x^{k} g_{k}(t)\right)$, or

$$
\sigma_{i}\left(\sum_{i}^{m} x^{k} g_{k}(t)\right)=u_{i}+\frac{1}{2} \sum_{i}^{m} x^{k}\left[g_{k}(t), u_{i}\right]+\sum_{n=1}^{\infty} \frac{(-1)^{n-1}}{2 n !} b_{n}\left(\operatorname{ad} \sum_{1}^{m} x^{k} g_{k}(t)\right)^{2 n} \cdot u_{i}
$$


Le coefficient de $x^{m}$ dans $\left(\operatorname{ad} \sum_{1}^{m} x^{k} g_{k}(t)\right)^{2 n}$ est, en utilisant l'expression des $g_{k}(t)$ obtenue au a) pour $k \leqq m$ :

$$
\sum_{D_{2 n}} B_{t}^{L_{1}} \ldots B_{t}^{L_{2 n}} \text { ad } \beta_{L} \ldots \text { ad } \beta_{L_{2 n}}
$$

où

$$
D_{2 n}=\left\{\mathscr{L}=\left(L_{1}, \ldots, L_{2 n}\right) / L_{i} \in \bigcup_{i}^{\infty}\{0 \ldots r)^{k} \text { et } \sum_{i=1}^{2 n}\left\|L_{i}\right\|=m\right\}
$$

à savoir, en utilisant l'expression des $\beta_{L_{i}}$ :

$$
\sum_{\mathscr{L} \in D_{2 n}} \sum_{Q \in E(\mathscr{L})} \frac{B_{t}^{L_{1}} \ldots B_{t}^{L_{2 n}}}{\left|L_{1}\right| \ldots\left|L_{2 n}\right|} \frac{(-1) \sum_{1}^{2 n} k\left(q_{i}\right)}{k(q(1) \ldots k(q(2 n))} \operatorname{ad} U_{L_{1}}^{q(1)} \ldots \operatorname{ad} U_{L_{2 n}}^{q(2 n)}
$$

où

$$
E(\mathscr{L})=\left\{Q=(q(1), \ldots, q(2 n)) ; q(i) \in \sum_{k=1}^{\left|L_{i}\right|} \widehat{B}_{k}\right\}
$$

Or, la formule d'Ito appliquée au produit montre que:

$$
B_{t}^{L_{1}} \ldots B_{t}^{L_{2 n}}=\sum_{\substack{J \\ \text { tels que } \\ \mathscr{L} \in F_{2 n}(J)}} B_{t}^{J}
$$

avec $F_{2 n}(J)=\left\{\mathscr{L}=\left(L_{1}, \ldots, L_{2 n}\right) \in D_{2 n}\right.$; les $L_{i}$ forment une partition de $J$ et chaque $L_{i}$ est une suite extraite de $\left.J\right\}$.

D'où l'on montre que l'expression (*) vaut:

$$
\sum_{\| J||=m} B_{t}^{J}\left(\sum_{\mathscr{L} \in F_{2 n}(J)} \sum_{Q \in E(\mathscr{L})} \frac{(-1)^{\Sigma k(q(l))}}{\left|L_{1}\right| \ldots\left|L_{2 n}\right| k(q(1) \ldots k(q(2 n))} \operatorname{ad} U_{L_{1}}^{q(1)} \ldots \text { ad } U_{L_{2 n}}^{q(2 n)}\right)
$$

et donc que le coefficient $C_{i}^{m}\left(g(t), \ldots, g_{m}(t)\right)$ cherché vaut:

$$
\sum_{D_{2 n}} B_{t}^{L_{1}} \ldots B_{t}^{L_{2 n}} \text { ad } \beta_{L} \ldots \text { ad } \beta_{L_{2} n}
$$

où

$$
D_{2 n}=\left\{\mathscr{L}=\left(L_{1}, \ldots, L_{2 n}\right) / L_{i} \in \bigcup_{i}^{\infty}\{0 \ldots r\}^{k} \text { et } \sum_{i=1}^{2 n}\left\|L_{i}\right\|=m\right\}
$$


à savoir, en utilisant l'expression des $\beta_{L_{i}}$ :

$$
\sum_{\mathscr{L} \in D_{2 n}} \sum_{Q \in E(\mathscr{L})} \frac{B_{t}^{L_{1}} \ldots B_{t}^{L_{2 n}}}{\left|L_{1}\right| \ldots\left|L_{2 n}\right|} \frac{(-1) \sum^{2 n} k\left(q_{i}\right)}{k(q(1) \ldots k(q(2 n))} \operatorname{ad} U_{L_{1}^{q(1)} \ldots \operatorname{ad} U_{L_{2 n}}^{q(2 n)} .}^{1}
$$

où

$$
E(\mathscr{L})=\left\{Q=(q(1), \ldots, q(2 n)) ; q(i) \in \sum_{k=1}^{\left|L_{i}\right|} \hat{B}_{k}\right\}
$$

Or, la formule d'Ito appliquée au produit montre que:

$$
B_{t}^{L_{1}} \ldots B_{t}^{L_{2 n}}=\sum_{\substack{J \\ \text { tels que } \\ \mathscr{L} \in F_{2 n}(J)}} B_{t}^{J}
$$

avec $F_{2 n}(J)=\left\{\mathscr{L}=\left(L_{1}, \ldots, L_{2 n}\right) \in D_{2 n}\right.$; les $L_{i}$ forment une partition de $J$ et chaque $L_{i}$ est une suite extraite de $\left.J\right\}$.

D'où l'on montre que l'expression $(*)$ vaut:

$$
\sum_{\|J\|=m} B_{t}^{J}\left(\sum_{\mathscr{L} \in F_{2 n}(J)} \sum_{Q \in E(\mathscr{L})} \frac{(-1)^{\Sigma k(q(l))}}{\left|L_{1}\right| \ldots\left|L_{2 n}\right| k(q(1) \ldots k(q(2 n))} \operatorname{ad} U_{L_{1}}^{q(1)} \ldots \operatorname{ad} U_{L_{2 n}}^{q(2 n)}\right)
$$

et donc que le coefficient $C_{i}^{m}\left(g(t), \ldots, g_{m}(t)\right)$ cherché vaut:

$$
\sum_{\|J\|=m} B_{t}^{J}\left\{\frac{1}{2}\left[\beta_{J}, u_{i}\right]+\sum_{n=1} \frac{(-1)^{n-1}}{2 n !} b_{n}\right.
$$

$$
\left[\sum_{\left.\sum_{\mathscr{L} \in F_{2 n}(J)} \sum_{Q \in E(\mathscr{L})} \frac{(-1)^{\Sigma k(q(l))}}{\left|L_{i}\right| \ldots\left|L_{2 n}\right| k(q(1) \ldots k(q(2 n)} \text { ad } U_{L_{1}}^{q(1)} \ldots \text { ad } U_{L_{2 n}}^{q(2 n)}\right]} \cdot U_{i} .\right.
$$

Il reste à vérifier que l'expression entre accolades est égale à $\beta_{J \cup\{i}$. Pour cela, remarquons que, si l'on note $H_{1}(u, v)$ la somme des termes bihomogènes de la série $H(u, v)$ dont le degré en $v$ est 1 , on a:

$$
\sigma_{i}(x)=H_{1}\left(x, u_{i}\right) \quad \text { (cf. Bourbaki [5]). }
$$

Par définition, $\beta_{J \cup\{i\}}$ est le coefficient de $x_{1} \ldots x_{m} y$ dans la série $H\left(x_{1} u_{j_{1}}, \ldots, x_{m} u_{j m}, y u_{i}\right)$ et donc celui de $x_{1} \ldots x_{m} y$ dans la série $H_{1}\left(H\left(x_{1} u_{j_{1}}, \ldots, x_{m} u_{j m}\right), y u_{i}\right)$, à savoir, dans la série:

$$
y u_{i}+\frac{1}{2}\left(x_{1} \ldots x_{m} y\right)\left[\beta_{J}, u_{i}\right]+y \sum_{n=1}^{\infty} \frac{(-1)^{n-1}}{2 n !} b_{n}\left[\operatorname{ad} H\left(x_{1} u_{j_{1}}, \ldots, x_{m} u_{j m}\right)\right]^{2 n} \cdot U_{i} .
$$


Grace à l'expression de la série $H\left(x_{1} u_{j_{1}}, \ldots, x_{m} u_{j m}\right)$ on vérifie que ce coefficient $\beta_{J} \cup\{1\}$ est donc égal à

$$
\begin{aligned}
& \frac{1}{2}\left[\beta_{J}, u_{i}\right]+\sum_{n=1}^{\infty} \frac{(-1)^{n-1}}{2 n !} b_{n} \\
& \cdot \sum_{P \in C_{2 n}} \frac{(-1)^{\Sigma k(p(l))}}{|p(1)| \ldots|p(2 n)| k(p(1) \ldots k(p(2 n))} \text { ad } U_{J}^{p(1)} \ldots \operatorname{ad} U_{J}^{p(2 n)} \cdot u_{i}
\end{aligned}
$$

où

$$
\begin{aligned}
C_{2 n}=\{ & P=(p(1), \ldots, p(2 n)) ; \quad p(l) \in \sum_{k=1}^{|J|} B_{k} ; \quad \forall i, l \sum_{j=1}^{k(p)} p(l)_{i}^{j} \leqq 1 ; \\
& \left.\forall i \exists !(l, j) p(l)_{i}^{j}=1\right\} .
\end{aligned}
$$

Nous allons vérifier que les expressions (A) et (B) coïncident, en introduisant (à $J$ fixé) une bijection entre $C_{2 n}$ et $G_{2 n}(J)$ où

$$
G_{2 n}(J)=\left\{(\mathscr{L}, Q), \mathscr{L} \in F_{2 n}(J), Q \in E(\mathscr{L})\right\} .
$$

Soit $P=(p(1), \ldots, p(2 n)) \in C_{2 n}$ et soit $l \in\{1 \ldots 2 n\}$ on a alors:

$$
\text { card }\left\{i, \exists j p(l)_{i}^{j} \neq 0\right\}=|p(l)| \text {. }
$$

Soit $\left(n_{1}, \ldots, n_{\mid p(l)}\right)$ l'ensemble $\left\{i, \exists j p(l)_{i}^{j} \neq 0\right\}$ récordonné de façon croissante.

Posons alors $L_{l}=\left(j_{n_{1}}, \ldots, j_{n|p(l)|}\right)$

$$
\text { et } q(l)_{i}^{j}=p(l)_{n_{i}}^{j} .
$$

On vérifie simplement que $\mathscr{L}=\left(L_{1}, \ldots, L_{2 n}\right) \in F_{2 n}(J)$

$$
\text { et que } Q=(q(1), \ldots, q(2 n)) \in E(\mathscr{L})
$$

et que, de plus

$$
\left.\begin{array}{rl}
\left|L_{l}\right| & =|p(l)| \\
k(p(l)) & =k(q(l)) \\
U^{p(l)} & =U_{L_{l}}^{q(l)} .
\end{array}\right\}
$$

L'application ainsi construite $C_{2 n} \rightarrow G_{2 n}(J)$ est une bijection

$$
P \rightarrow(\mathscr{L}, Q) .
$$

En effet, on peut construire ainsi son inverse:

Si $(\mathscr{L}, Q) \in G_{2 n}(J)$ et si $l \in\{1 \ldots 2 n\}$ alors si $\mathscr{L}=\left(L_{1}, \ldots, L_{2 n}\right)$ on sait que $L$ est une suite extraite de $J$; notons la $\left(j_{n_{1}} \ldots j_{n_{k}}\right)$. Posons alors:

$$
p(l)_{i}^{\dot{j}}=0 \quad \text { si } i \notin\left\{n_{1}, \ldots, n_{k}\right\}
$$

et $p(l)_{n_{i}}^{j}=q(l)_{i}^{j} \quad$ sinon. 
Il est alors facile de vérifier que $P=(p(1), \ldots, p(2 n))$ définit un élement de $C_{2 n}$ et que l'application qui, à $(\mathscr{L}, Q)$, associe $P$ est l'inverse de l'application précédente, qui est donc bijective. Cette remarque et les relations (**) montrent que:

$$
\begin{aligned}
& \sum_{p \in C_{2 n}} \frac{(-1)^{\Sigma k(p(l))}}{|p(1)| \ldots \mid p(2 n \mid k(p(1)) \ldots k(p(2 n))} \text { ad } U_{J}^{p(1)} \ldots \text { ad } U_{J}^{p(2 n)} \cdot u_{i} \\
& \quad=\sum_{\mathscr{L} \in F_{2 n}(J)} \sum_{Q \in E(\mathscr{L})} \frac{(-1)^{\Sigma k(q(l))}}{\left|L_{1}\right| \ldots\left|L_{2 n}\right| k(q(1)) \ldots k(q(2 n))} \text { ad } U_{L_{1}}^{q(1)} \ldots \text { ad } U_{L_{2 n}}^{q(2 n)} \cdot u_{i}
\end{aligned}
$$

et donc que les expressions (A) et (B) coïncident. Ce qui achève la preuve, du fait que:

$$
C_{i}^{m}\left(g_{1}(t), \ldots, g_{m}(t)=\sum_{|J| \|=m} \beta_{J \cup\{i\}} B_{t}^{J}\right.
$$

et donc, par récurrence, la preuve du lemme. Ce qui, à son tour, achève la preuve du théorème, puisque le lemme donne l'expression explicite cherchée de la série de Taylor stochastique de $\zeta_{t}$.

\section{Bibliographie}

1. Azencott, R.: Formule de Taylor stochastique et développements asymptotiques d'intégrales de Feynmann. In: Azema, J., Yor, M. (eds.) Séminaire de probabilités XVI (Lect. Notes Math., vol. 921, pp. 237-284) Berlin Heidelberg New York: Springer 1982

2. Azencott, R.: Densités des diffusions en temps petit: developpements asymptotiques. In: Séminaire de probabilités XVIII. (Azema, J., Yor, M. (eds.) (Lect. Notes Math., vol. 1059, pp. 402-498). Berlin Heidelberg New York: Springer 1984

3. Berger, M., Gauduchon, P., Mazet, E.: Le spectre d'une variété riemanienne. (Lect. Notes Math., vol. 194). Berlin Heidelberg New York: Springer 1971

4. Bismut, J.M.: Mecanique alétoire. (Lect. Notes Math., vol. 866). Berlin Heidelberg New York: Springer 1981

5. Bourbaki, N.: Groupes et algèbres de Lie, tome 2. Paris: Masson 1972

6. Doss, H.: Lien entre équations différentielles stochastiques et ordinaires. Ann. Inst. Henri Poincare, Novv. Ser., Sect. B 13, 99-125 (1977)

7. Fliess, M., Normand-Cyrot, D.: Algèbres de Lie nilpotentes, formule de Baker-Campbell-Hausdorff et intégrales itérées de K.T. Chen. In: Azema, J., Yor, M. (eds.) Seminaire de probabilités XVI (Lect. Notes Math., vol. 920, pp. 257-267). Berlin Heidelberg New York: Springer 1982

8. Gaveau, B.: Principe de moindre action, propagation de la chaleur et estimées sous elliptiques sur certains groupes nilpotents. Acta Math. 139, 95-153 (1977)

9. Ibero, M.: Intégrales stochastiques multiplicatives. Bull. Sci. Math. 100, 175-191 (1976)

10. Kunita, H.: On the decomposition of solutions of stochastic differential equations. In: Williams, D. (red.) Proceedings, LMS Durham Symposium, 1980. Lect. Notes Math., vol. 851, pp. 213-284). Berlin Heidelberg New York: Springer 1981

11. Kunita, $H$.: On the representation of solutions of stochastic differential equations. Séminaire de probabilités XIV. In: Azema, J., Yor; M. (eds.), (Lect. Notes Math., vol. 784, pp. 282-304. Berlin Heidelberg New York: Springer 1980

12. Malliavin, P.: Parametrix trajectorielle pour un opérateur hypoelliptique et repère mobile stochastique. C.R. Acad. Sci., Paris, Ser. I 281, 241 (1975)

13. Malliavin, P.: Géométrie différentielle stochastique. Montréal: Presses de l'université de Montréal 1978

14. Meyer, P.A.: Cours sur l'intégrale stochastique. In: Meyer, P.A. (ed.) Séminaire de probabilités X. (Lect. Notes Math., vol. 511, pp. 321-331). Berlin Heidelberg New York: Springer 1976 
15. Palais, R.: A global formulation of the Lie theory on transformation groups. Mem. Am. Math. Soc. 22, 95-97 (1957)

16. Platen, E.: A Taylor formula for semimartingales solving a stochastic equation. In: Third conference on stochastic differential systems, pp. 65-68. Visegrad: Hongrie 1980

17. Sussmann, H.: On the gap between deterministic and stochastic ordinary equations. Ann. Probab. 6, 19-41 (1978)

18. Yamato, Y.: Stochastic differential equations and nilpotent Lie algebras. Z. Wahrscheinlichkeitstheor. Verw. Geb. 47, 213-229 (1979)

19. Krener, A.J., Lobry, C.: The complexity of stochastic differential equations. Stochastics 4, 193-203 (1981)

20. Abraham, R., Marsden, J., Ratiu, T.: Manifolds, tensor analysis and applications. Reading, Mass.: Addison Wesley 1983

21. Nagano, T.: Linear differential systems with singularities and applications to transitive Lie algebras. J. Math. Soc. Japan 18, 398-404 (1966)

Received March 10, 1986; in revised form March 14, 1988 